\title{
Precision studies of deep-inner-shell photoabsorption by atomic ions
}

\author{
A. Müller \\ Institut für Atom- und Molekülphysik, Justus-Liebig-Universität Giessen, Germany \\ E-mail: Alfred.Mueller@iamp.physik.uni-giessen.de
}

\begin{abstract}
With emphasis on $K$-shell ionization and $K$-shell excitation mediated by the absorption of a single photon the present paper provides an overview of the status of experiments in which deep inner-shell photoabsorption by atomic ions has been investigated. For inner-shell processes not involving the $K$-shell only photon energies beyond about $350 \mathrm{eV}$ are considered. Mechanisms of photoabsorption and subsequent relaxation as well as relations between photoionization and photorecombination are discussed. New possibilities for precise measurements of transition energies have been provided by the high photon-energy resolution available at $3^{\text {rd }}$ generation synchrotron light sources. This is particularly interesting for studies of $K$-shell excitation of fewelectron systems. By using atomic ions as targets, either in form of a collimated beam or trapped in electromagnetic fields, it has been possible to reach a high level of precision in the spectroscopy of transitions involving the decay of $K$ vacancies by photoemission or Auger processes. Examples for absolute cross section measurements and precision spectroscopy are presented.
\end{abstract}

PACS numbers: 32.80.Aa,32.80.Fb,32.80.Hd,32.80.Zb

\section{Introduction}

Extensive atomic data are required to estimate opacities of stellar envelopes and interstellar gases. Important data needs have been addressed by theoretical calculations within the Opacity and the Iron Projects [1,2]. With the launch of satellite-borne high-resolution x-ray spectrometers (XMM-Newton, CHANDRA) which are employed to study hot astrophysical environments such as the vicinity of supermassive black holes, strong interest in opacity data has evolved towards inner-shell photoabsorption processes [3] in ionized matter.

Pioneering work on inner-shell photoabsorption has used plasma techniques to both produce short-wavelength radiation and to provide targets containing ions at high densities. The history of such experiments has been reviewed by Kennedy et al [4]. First observations of $K$-shell photoabsorption were made for $\mathrm{Be}^{+}$ions [5] using two vacuumsparc discharges. Two laser-produced plasmas were employed to investigate $K$-shell absorption of heliumlike $\mathrm{Li}^{+}[6,7,8]$. Due to the high column density of the absorbing 
ion species, though mixed with other charge states, the total absorption spectrum could be measured in the dual-plasma-based experiments. Measured yields were normalized to theory and the energy resolving power in these experiments was limited to about 1500 .

The construction of suitable experimental arrangements and the development of synchrotron light sources during the last 15 years has facilitated new, more precise experiments studying deep-inner-shell photoabsorption by atomic ions. Photoexcitation and -ionization involving the $K$ shell were explored for $\mathrm{O}^{+}$ions [9] and $\mathrm{Ne}^{q+}$ $(q=1,2,3)$ ions [10, 11] at SPRING-8. Photoionization via $K$-shell excitation of $\mathrm{Li}^{+}[12]$, $\mathrm{B}^{+}[13], \mathrm{B}^{2+}[14], \mathrm{C}^{+}[15], \mathrm{C}^{2+}[16]$, and $\mathrm{C}^{3+}$ [17] has been measured at the Advanced Light Source ALS. More recently, a new experimental photon-ion experiment became operational at SOLEIL and $K$-shell excitation with subsequent single autoionization was studied for $\mathrm{N}^{+}$[18], $\mathrm{N}^{2+}$ [19], $\mathrm{N}^{3+}$ [20], $\mathrm{N}^{4+}$ [20], and $\mathrm{O}^{3+}$ [21]. In a series of experiments at the ALS photo-double-detachment of negative atomic ions $\mathrm{He}^{-}[22,23]$, $\mathrm{Li}^{-}$[24], $\mathrm{B}^{-}$[25] and $\mathrm{C}^{-}$[26] was measured near the respective $K$ edges. Prior to these experiments absolute cross sections for near- $K$-edge photo-double-detachment of $\mathrm{Li}^{-}$ had been published by a group from Aarhus employing the photon-ion merged-beam facility at the storage ring ASTRID [27]. Work on photo detachment of $\mathrm{H}^{-}$and $\mathrm{D}^{-}$ dates back much further since Doppler-tuned laser light instead of synchrotron radiation could be employed to excite "inner-shell" resonances. The status of research on hydrogen anions until the end of 2003 was reviewed by Andersen [28]. The most recent results in this context were published on Doppler-tuned VUV spectroscopy of few-MeV Dions [29]. For negative hydrogen ions the concept of an electron initially residing in the $K$-shell is not adequate. Moreover, the resonance energies are naturally only in the range between about 10 and $14 \mathrm{eV}$. Therefore, this wide field of research is not considered further in the present article.

New possibilities for studying deep-inner-shell photoabsorption were opened up by combining an electron beam ion trap (EBIT) with intense photon beams of shortwavelength free-electron lasers (FEL) or synchrotron radiation and observing radiative or Auger decay processes after inner-shell excitation. First experiments with an EBIT were carried out at FLASH, the free-electron laser in Hamburg, and at BESSY in Berlin covering outer-shell photoexcitation. These measurements were soon extended to the region of inner shells of $\mathrm{Ar}^{12+}$ and $\mathrm{Fe}^{12+}$ [30], of $\mathrm{Ar}^{8+}$ [31], of $\mathrm{Fe}^{14+}$ [32] and, at the Linac Coherent Light Source LCLS, of $\mathrm{Fe}^{16+}[33]$. More recently, the EBIT photoabsorption measurements were extended to $K$-shell excitation of highly charged iron ions from heliumlike to fluorinelike $\left(\mathrm{Fe}^{17+}\right.$ to $\mathrm{Fe}^{24+}$ ) in the energy region around $6.6 \mathrm{keV}$ using monochromatized $x$ rays from the PETRA III synchrotron photon source [34]. In these experiments relative yields of the main $K_{\alpha}$ transitions were determined by observing the fluorescence radiation. Additionally, the ion inventory of EBIT after exposure to the PETRA III synchrotron radiation was extracted and charge-analyzed such as to obtain absolute radiative and Auger decay rates for Li-like, Be-like, B-like and C-like iron ions [35] subsequent to $K$-shell photoabsorption. The most highly charged ion 
addressed so far in such experiments is $\mathrm{Kr}^{34+}$ [36]. The measurements permitted highprecision determination of the transition energies from the $1 s^{2}{ }^{1} S_{0}$ ground state to the $1 s 2{ }^{1} P_{1}$ (w line) and $1 s 2 p^{3} P_{1}$ (y line) excited states in heliumlike krypton by resonant single-photon absorption.

All the merged-beam or EBIT experiments mentioned above are restricted to the strongest resonances and never covered complete spectra from the lowest-energy $K$-shell excitations to and beyond the $K$-shell ionization threshold. Observation channels comprise single ionization associated with $K$-shell excitation, studied by detecting photo-ions, and fluorescence following $K$-shell excitation, studied by detecting resonantly scattered photons. Absolute cross sections were primarily (but not always) determined in the merged-beam experiments.

A breakthrough was recently accomplished at the newly constructed setup PIPE, the Photon-Ion Spectrometer at PETRA III, which is an experimental facility for studying interactions of single photons with charged particles (atomic and molecular ions, electrically charged clusters and nanoparticles) [37]. It has been set up at the Variable Polarization XUV beamline P04 at PETRA III which is presently the brightest synchrotron radiation source worldwide. Compared to previous work the range of possible measurements could be greatly extended.

For $\mathrm{Xe}^{q+}$ ions with $q=1, \ldots, 5$ in the $3 d$ ionization threshold range [38] as well as for $\mathrm{C}^{q+}$ ions with $q=1,2,3,4$ [39] and for $\mathrm{Ne}^{q+}$ ions with $q=1,2,3$ [40] in the $K$-edge energy region absolute cross sections for photoionization by single photons were measured with resolving powers $E / \Delta E$ reaching up to almost 40,000. Different from all previous photoionization and photoexcitation experiments with ions other than $\mathrm{He}^{+}$and $\mathrm{Li}^{+}$, the whole energy range around the $K$ edge could be covered from the lowest-energy resonances involving $K$-shell excitation to beyond the $K$-shell ionization threshold including double excitations of both a $K$ - and an $L$-shell electron. Moreover, not only single ionization but also multiple ionization could be addressed in photon-ion $K$-shell experiments for the first time. A large window to deep-inner-shell photoabsorption spectroscopy of ions has been opened providing unprecedented access to new territory of research on photoabsorption and subsequent decay processes. For example, by the choice of individual photoexcitation resonances in a five-electron ion, $\mathrm{C}^{+}$, first unambiguous evidence was found for a four-electron decay mechanism, the triple-Auger process, in which one electron drops from the $L$ shell into a $K$ vacancy while all three remaining $L$-shell electrons are simultaneously ejected into the continuum [41].

\section{Mechanisms}

Photoabsorption is often associated with direct photoionization as evidenced by the x-ray attenuation data provided by the US National Institute for Standards and Technology (NIST) under http://physics.nist.gov/PhysRefData/XrayMassCoef/tab3.html. However, there is a large number of additional mechanisms (sometimes quite exotic) by which atoms or ions can absorb photons. An overview of some of these mecha- 


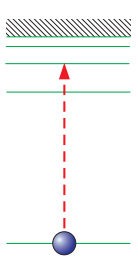

(a)

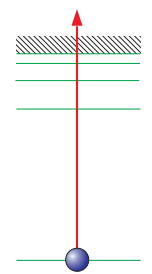

(b)

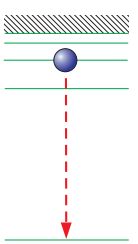

(c)

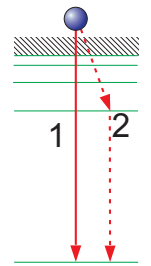

(d)

Figure 1. (Colour online) Photoabsorption by a hydrogenlike atom or ion and the associated time-reversed processes; (a) photoexcitation, (b) photoionization, (c) radiative decay, (d) photorecombination (path 1 leads to the ground-state of a hydrogenlike atom or ion, path 2 proceeds via capture into an excited state and subsequent radiative relaxation to the ground state.

nisms is provided in this section showing the physical background of results presented or discussed later.

The structurally simplest absorbers are one-electron systems. Fig. 1 shows the two possible groups of photoabsorption mechanisms. These are photoexcitation (Fig. 1(a)) to a bound level and photoionization (Fig. 1(b)) leaving the initially bound electron in the continuum. In the particular case of hydrogenlike parent atoms or ions the only possible relaxation of an excited state is via emission of radiation. This can occur by the time-reversed excitation back to the initial state (Fig. 1(c)) or via a cascade of radiative transitions (not shown in Fig. 1). The time-inverse of photoionization is photorecombination. In the present context, time reversal of the process shown in Fig. 1(b) results in the recombination mechanism represented by path 1 in Fig. 1(d). Of course, recombination can also proceed through a multi-step process consisting of radiative recombination to a highly excited level with a subsequent cascade of radiative decays as indicated by path 2 .

The cross section $\sigma_{i k}\left(E_{\gamma}\right)$ for resonant excitation of an atom or ion by a single photon with energy $E_{\gamma}$ is closely related to the oscillator strengths $f_{i k}$ of the electronic transitions from the ground state $i$ to the excited states $k$ involved in the photoabsorption processes which contribute to the cross section. The absorption oscillator strength $f_{i k}$ can be inferred from the resonance strength $\bar{\sigma}_{i k}$ of the associated transition, that is, the peak area under the absorption resonance related to the transition $i \rightarrow k$

$$
\bar{\sigma}_{i k}=\int_{E_{\gamma}} \sigma_{i k}\left(E_{\gamma}\right) d E_{\gamma} .
$$

The oscillator strength is then

$$
f_{i k}=\frac{\bar{\sigma}_{i k}}{4 \pi^{2} \alpha a_{0}^{2} \mathcal{R}},
$$

with the fine-structure constant $\alpha$, the first Bohr radius $a_{0}$ and the Rydberg energy $\mathcal{R}$. The oscillator strength $f_{i k}$ for an electric dipole transition $i \rightarrow k$ can also be expressed 
by its dependence on the transition rate $A_{k i}$ for the radiative decay $k \rightarrow i$

$$
f_{i k}=1.4992 \times 10^{-14} \lambda_{k i}^{2} \frac{g_{k}}{g_{i}} A_{k i},
$$

where $\lambda_{k i}$ is the wavelength of the transition photon in units of $\mathrm{nm}, g_{k}$ and $g_{i}$ are the statistical weights of states $k$ and $i$, respectively, and $A_{k i}$ is in units s ${ }^{-1}$. The sum of all transition rates associated with decays $k \rightarrow f$ to all possible final states determines the radiative decay width $\Gamma_{k}^{(r)}$ of the resonant state $k$

$$
\sum_{f} A_{k f}=\Gamma_{k}^{(r)} / \hbar
$$

Hence, the absorption cross section $\sigma_{i k}$ resulting from $i \rightarrow k$ excitation of a nonautoionizing state $k$ can be written as

$$
\sigma_{i k}\left(E_{\gamma}\right)=f_{i k} 4 \pi^{2} \alpha a_{0}^{2} \mathcal{R} \frac{1}{2 \pi} \frac{\Gamma_{k}}{\left(E_{\gamma}-E_{i k}\right)^{2}+\frac{\Gamma_{k}^{2}}{4}} .
$$

$E_{i k}$ is the energy of the $i \rightarrow k$ transition and, in the particular case of a hydrogenlike ion, $\Gamma_{k}=\Gamma_{k}^{(r)}$ is the total width of the excited state $k$.

The energy-dependent cross section for photoionization of a hydrogenlike atom or ion (Fig. 1(b)) was already calculated with quite good accuracy at the dawn of quantum mechanics [42]. Using non-relativistic wave functions Stobbe succeeded to develop closed formulas for cross sections describing the ionization of a hydrogen atom (or a hydrogenlike ion) from an initial state $i$ to the continuum. For the $1 s^{2} S$ ground state of a hydrogenlike system the photoionization cross section can be represented as $[42,43]$

$$
\sigma_{P I}^{1 s}=\frac{2^{6} \alpha h^{2} I^{3} e^{-4 \nu \arctan \left(\frac{1}{\nu}\right)}}{3 m_{e} E_{\gamma}^{4}\left(1-e^{-2 \pi \nu}\right)},
$$

where $h$ is Planck's constant, $I$ the ionization potential of the $1 s$ ground state, $m_{e}$ the electron rest mass, and $\nu$ the Sommerfeld parameter

$$
\nu=\sqrt{\frac{I}{E_{\gamma}-I}} .
$$

More modern, fully relativistic calculations [44] agree remarkably well with the Stobbe result for the hydrogen atom. For an estimate of cross sections for direct $K$-shell photoionization of more complex atoms or ions equation 6 can be used with the related ionization potential $I$ and by multiplying the cross section $\sigma_{P I}^{1 s}$ by the actual number of electrons residing in the $K$ shell, i. e., normally 2. Since Stobbe also derived similar formulas for the other $n l$ subshells of H-like systems, the total photoionization cross section of any given atom can be reasonably well approximated accordingly. For each subshell a function is obtained that jumps to its maximum at threshold and then falls off with increasing photon energy approximately decreasing with $1 / E_{\gamma}^{7 / 2}$.

The process depicted in Fig. 1(d) (path 1) is time reversed with respect to $1 \mathrm{~s}$ photoionization. Time reversal symmetry and the principle of detailed balance (or micro-reversibility) [45] relate cross sections $\sigma_{P I}\left(E_{\gamma}\right)$ for photoionization

$$
\gamma+|a\rangle \rightarrow|b\rangle+e-I_{b i n d}
$$




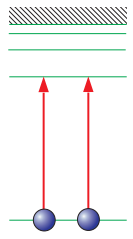

(a)

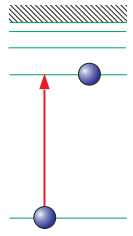

(b)

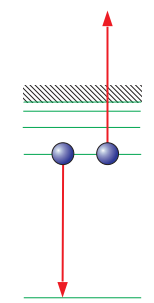

(c)

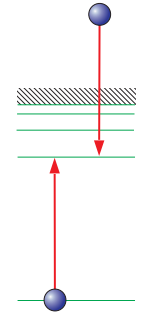

(d)

Figure 2. (Colour online) Photoabsorption by a two-electron atom or ion and timereversed processes; (a) double excitation of the ground state of a heliumlike system, (b) single excitation of a $K$-shell excited He-like atom or ion, (c) Auger decay of the intermediate state produced by (a) or (b), (d) time-reversed Auger decay $=$ dielectronic capture of a free electron into a hydrogenlike system.

and cross sections $\sigma_{P R}\left(E_{e}\right)$ for (photo)recombination

$$
e+|b\rangle \rightarrow|a\rangle+\gamma+I_{b i n d}
$$

on a level-to-level basis $(|a\rangle \rightarrow|b\rangle$ and $|b\rangle \rightarrow|a\rangle$ with well defined levels $|a\rangle$ and $|b\rangle)$. The binding energy $I_{b i n d}$ is the difference of the total energies of the final and the initial levels $I_{\text {bind }}=E(|b\rangle)-E(|a\rangle)$. The energy of the photoelectron (in the electron-ion center-of-mass system) is $E_{e}=E_{\gamma}-I_{\text {bind }}$. The principle of detailed balance [45] yields the following relation for nonrelativistic photon energies $h \nu \ll m_{e} c^{2}$

$$
\frac{\sigma_{P R}^{b \rightarrow a}}{\sigma_{P I}^{a \rightarrow b}}=\frac{g_{a}}{g_{b}} \frac{E_{\gamma}^{2}}{2 m_{e} c^{2} E_{e}}
$$

where the quantities $g_{a}$ and $g_{b}$ are the statistical weights of the initial and final states as given by Eqs. 8 and 9, respectively. Thus, from Eqs. 10 and 6 one can immediately calculate the cross section $\sigma_{P R}\left(E_{e}\right)$ for the photorecombination process of a completely ionized ion to the $1 s$ ground level of the hydrogenlike ion as represented by path 1 in Fig. 1(d).

When the absorber is heliumlike new additional mechanisms for absorbing a photon and for subsequent decay and time-reversed processes beyond the reaction pathways of Fig. 1 have to be considered. The most probable new pathways are illustrated in Fig. 2. It is possible now to simultaneously excite (Fig. 2 (a)) or photoionize (not shown in Fig. 2) both electrons in the system. By exciting both electrons, configurations are populated that can autoionize via Auger decays (Fig. 2 (c)). Such autoionizing configurations can also be reached from a core-excited heliumlike system (e.g., in a metastable $1 s 2 s$ configuration) via $K$-shell excitation of the $1 s$ core electron (Fig. 2 (b)). The Auger decay proceeds via electron-electron interaction in the doubly excited configuration ( $2 s 2 p$ in Fig. $2(\mathrm{c})$ ) with one electron falling back to the $K$ shell and the other being ejected to the continuum. The associated time-reversed autoionization as shown in Fig. 2 (d) is termed dielectronic capture (DC), a radiationless attachment of a free electron to an atomic ion, which constitutes the first step in resonant electron-ion recombination, generally termed dielectronic recombination (DR) [46]. DR is completed 


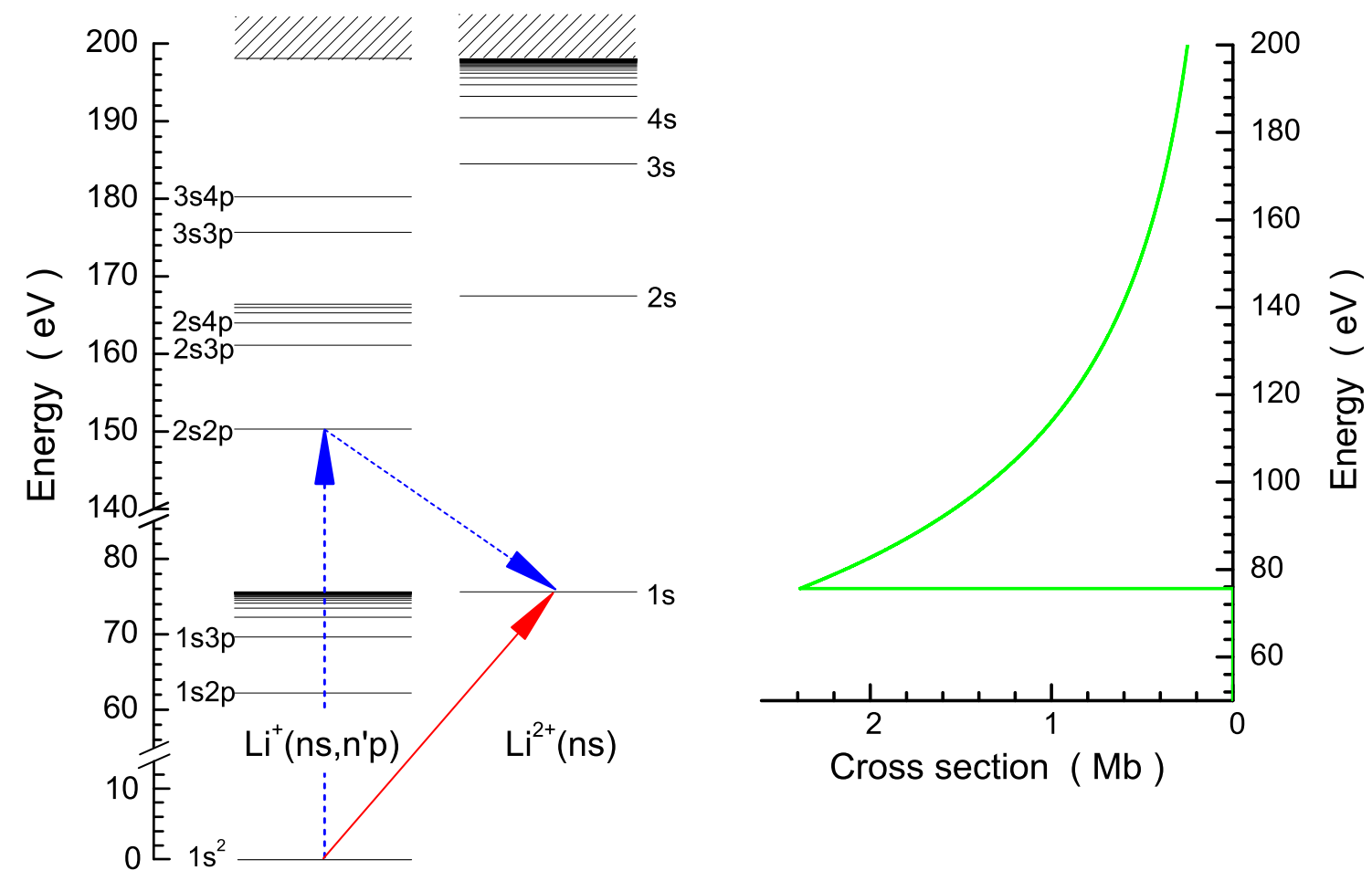

Figure 3. (Colour online) On the left a level diagram of a two-electron system is given. The energies are close to realistic values for the $\mathrm{Li}^{+}$ion. The solid (red online) arrow indicates one of the possible pathways to $\mathrm{Li}^{2+}$ products: direct photoionization. The dashed (blue online) arrows show a possible two-step path $1 s^{2} \rightarrow 2 s 2 p \rightarrow 1 s+e$ to $\mathrm{Li}^{2+}$ products: photo-double-excitation with subsequent Auger decay. On the right side the cross section for direct photoionization [47] of the $\mathrm{Li}^{+} K$-shell (corresponding to the process indicated by the solid arrow on the left) is shown with the energy axis corresponding to the range of levels on the left (without the break applied to the level diagram).

by radiative stabilization of the recombined (but highly excited and, hence, unstable) system populated by DC. Such radiative stabilization would be, for example, the timereverse of the excitation process depicted in Fig. 1 (c) (with an additional electron as a spectator).

Another new aspect of the photoionization of a target containing at least two electrons is the possibility that the amplitudes of different pathways leading to net ionization can interfere with one another. The situation is illustrated by Fig. 3 where an energy level diagram of He-like $\mathrm{Li}^{+}$is shown including some prominent singly and multiply excited configurations. Also shown is a simplified level diagram of the resulting $\mathrm{H}$-like $\mathrm{Li}^{2+}$ ion with its main electron shells. Direct ionization of $\mathrm{Li}^{+}\left(1 s^{2}\right)$ by a photon with energy $E_{\gamma}$ produces a $\mathrm{Li}^{2+}(1 s)$ photoion and a photoelectron that carries away the excess angular momentum and the energy $E_{\gamma}-I$ with the $K$-shell ionization threshold I. This path is represented by the solid (red) arrow. For the general case, it is described 


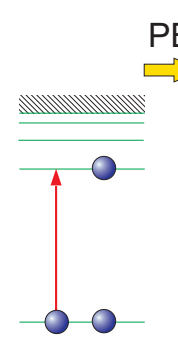

(a)

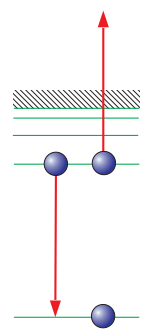

(b)

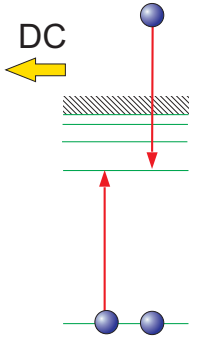

(c)

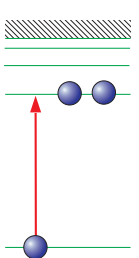

(d)

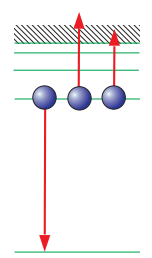

(e)

Figure 4. (Colour online) Photoabsorption by a lithiumlike atom or ion and subsequent electron-emission processes; (a) $K$-shell photoexcitation (PE) of a groundstate three-electron system, (b) Auger decay of the intermediate $K$-vacancy state, (c) dielectronic capture (DC) into a ground-state heliumlike system. Processes (a), (b) and (c) correspond to processes (b), (c), (d) of Fig. 2. Here, however, the parent atom or ion is in its ground state and a second $K$-shell electron is merely a spectator. The panels (d) and (e) illustrate a specific case with (d) photoexcitation of a three-electron system with a $K$ vacancy, and (e) subsequent double-Auger decay.

by

$$
\gamma+\mathrm{A}^{q+}\left(1 s^{2}\right) \rightarrow \mathrm{A}^{(q+1)+}(1 s)+e+\left(E_{\gamma}-I\right) .
$$

The cross section for direct $K$-shell ionization is similar to the prediction of Stobbe for H-like systems (multiplied by a factor 2 to account for the presence of two $K$-shell electrons. It is shown by the thick (green) line on the right panel of Fig. 3 for the energy range of the level diagram on the left. The cross section jumps to its maximum when the photon energy passes the ionization energy and then it falls off roughly with $E_{\gamma}^{-7 / 2}$.

A second possible pathway to the $\mathrm{A}^{(q+1)+}(1 s)$ photoion is associated with a two-step mechanism involving photo-double-excitation with subsequent autoionization

$$
\gamma+\mathrm{A}^{q+}\left(1 s^{2}\right) \rightarrow \mathrm{A}^{q+}\left(n s n^{\prime} p\right) \rightarrow \mathrm{A}^{(q+1)+}(1 s)+e+E_{\text {Auger }},
$$

with $n, n^{\prime} \geqq 2$. Such a path is indicated in Fig. 3 by the dashed (blue) arrows. When the photon energy matches the excitation energy for a $\left(n s n^{\prime} p\right)$ resonance then the energy of the resulting Auger electron is equal to the excess energy associated with direct photoionization $E_{\text {Auger }}=E_{\gamma}-I$. This means that the two pathways described by Eqs. 11 and 12 are indistinguishable and, hence, the associated amplitudes interfere with one another. The resulting resonance cross sections can strongly differ from the Lorentzian shape represented by Eq. 5 depending on the phase shift between the two amplitudes. 


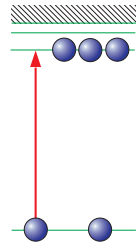

(a)

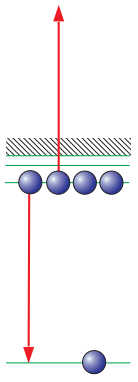

(b)

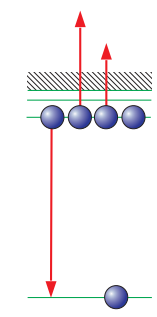

(c)

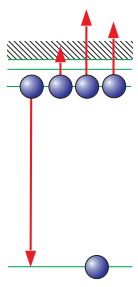

(d)

Figure 5. (Colour online) Photoabsorption by a boronlike atom or ion and subsequent electron emission processes; (a) $K$-shell photoexctation of a 5 -electron system, (b) single-Auger decay, c) double-Auger decay, and d) triple-Auger decay.

The shape of the resonance cross sections can be described by the formula [48]

$$
F\left(E_{\gamma}\right)=\frac{A}{Q^{2} \Gamma} \frac{2}{\pi}\left[\frac{(Q+\varepsilon)^{2}}{1+\varepsilon^{2}}-1\right]
$$

with $\varepsilon=2\left(E_{\gamma}-E_{\text {res }}\right) / \Gamma$, the resonance energy $E_{r e s}$, the resonance width $\Gamma$, and the asymmetry parameter $Q$.

When adding yet another electron to produce a lithiumlike absorber, again new mechanisms for one-photon absorption and associated decay processes contribute to the cross section for the specific observation channels. Single photoexcitation (PE) of a core electron in the ground state (see Fig. 4 (a)) already produces an autoionizing configuration (Fig. 4 (b)) and thus can strongly contribute to the net ionization of the target atom or ion which is experimentally observable by analyzing the final charge state of the absorber (usually a photoion). Again, the same configuration can be populated by DC (this time into a heliumlike ion as shown in Fig. 4 (c)). As before, the intermediate autoionizing state also has the possibility to decay radiatively which allows the experimenter to observe fluorescence light after PE or after DC. Triple ionization of a three-electron system (not shown in Fig. 4) by a single photon has been observed for Li atoms [49] but never for ions.

An exotic three-electron absorber is the $\mathrm{He}^{-}$ion with a configuration $1 s 2 s 2 p$ forming long-lived ${ }^{4} P$ states. Excitation of the $1 s$ electron (see Fig. $4(\mathrm{~d})$ ) produces a hollow configuration with no electrons in the $K$ shell. The associated resonance can decay by a number of mechanisms among which is a double-Auger process as illustrated in Fig. 4 (e). Double detachment of $\mathrm{He}^{-}$via this mechanism was observed by Bilodeau et al [22].

The main photoabsorption and decay mechanisms discussed so far can also be observed in many-electron ions. In order to illustrate the newly discovered tripleAuger decay mechanism one additional figure is added to this section. Fig. 5 shows photoexcitation (Fig. 5 (a)) and subsequent Auger processes in a five-electron system. Single-Auger (Fig. 5 (b), Ref. [50]) and double-Auger (Fig. 5 (b), Ref. [51]) processes have been known since many years. The sequence of Auger decay processes shown in 
Fig. 5 includes the triple-Auger decay in panel (d). This four-electron Auger process was recently demonstrated by Müller et al [41].

It is often cumbersome to find evidence of higher-order processes which typically have low probabilities and compete with overwhelming signals arising from first order mechanisms. A wise approach to unambiguously identify higher-order processes in an experiment is to restrict the number of electrons in the system to a minimum, such as to avoid additional pathways obscuring the desired effect. The minimum number of electrons required to observe triple-Auger decay in an atom is four. In the ideal case these four electrons are in the $L$-shell of a four-electron atomic system. Such a state can hardly be prepared via photoabsorption by a sufficiently long-lived atomic target. However, an additional electron in the $K$ shell merely acts as a spectator and does not complicate the situation. An electronic configuration with a $K$-shell vacancy and four electrons in the L-shell can be prepared by pure $K$-shell ionization (without additional excitation) of a C-like atom or ion. Alternatively, $K \rightarrow L$ photoexcitation of a B-like atom or ion $\mathrm{A}^{q+}$ with $q=-1,0,1,2, \ldots$ to a specific electronic level can be employed which has the advantage of a resonance signature. Since the final charge state after triple-Auger decay is $q+3$ the two electrons remaining after the decay process must be in the $K$ shell. Any cascade process would leave at least one electron in the $\mathrm{L}$ shell. Detecting the production of $\mathrm{A}^{(q+3)+}$ ions after $K$-shell excitation of B-like $\mathrm{A}^{q+}$ has the potential of unambiguously identifying the direct triple-Auger process. Candidates for such a demonstration are $\mathrm{Be}^{-}, \mathrm{B}, \mathrm{C}^{+}, \mathrm{N}^{2+}$ and so forth. Among these, seen from the experimental perspective, $\mathrm{C}^{+}$is the ion of choice to search for direct triple-Auger decay.

\section{Examples}

\subsection{H-like ions}

Photoprocesses in hydrogen atoms have been studied for two centuries. In particular, the precise measurements of wavelengths for photoexcitation and deexcitation of hydrogen have greatly contributed to the advancement of physics during all that time. Theory can now describe the structure and the decay properties of the hydrogen atom, and hydrogenic systems in general, extremely well. Issues under discussion involve, for example, such details as the size of the proton measured in muonic hydrogen [52], the distribution of the magnetic moment in nuclei $[52,53]$ and the precise determination of quantum-electrodynamic effects both in the hydrogen atom itself [54] and in hydrogenic ions with very heavy nuclei up to uranium [55, 56]. Experiments in which transition energies are determined for processes in hydrogen as shown in Fig. 1 (a) and (c) have provided results with relative uncertainties as low as some $10^{-15}$ for the $\mathrm{H}(1 \mathrm{~s}) \rightarrow \mathrm{H}(2 \mathrm{~s})$ transition [54]. This level of precision is presently far beyond the reach of experiments with ions. In contrast to that, photoabsorption experiments with the slightly more complex He-like ions, do presently start to push the precision for the determination of the $1 s^{2} \rightarrow 1 s 2 p$ transition wavelengths to a new frontier [34]. Photoprocesses of He-like 


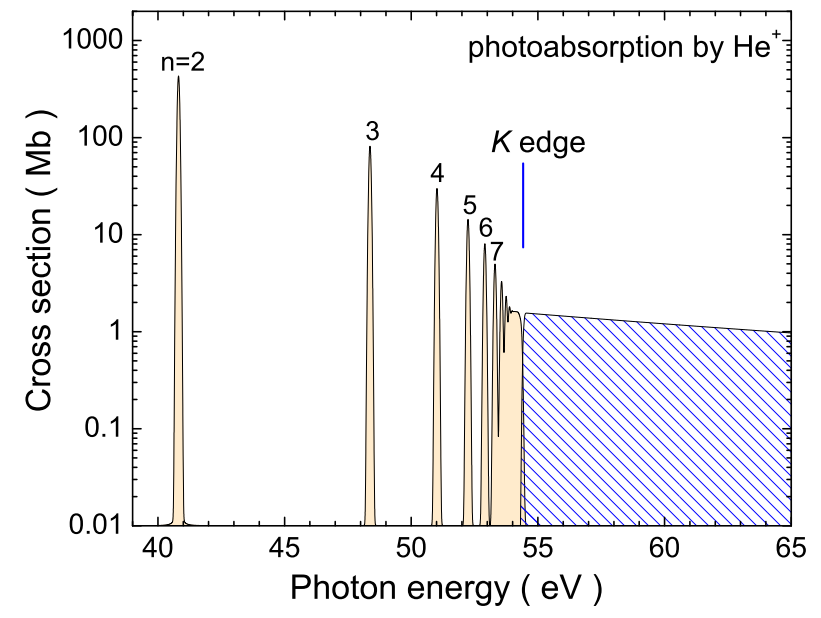

Figure 6. (Colour online) Calculated absorption cross section for hydrogenlike $\mathrm{He}^{+}$ ions. The solid vertical bar (blue online) indicates the ionization threshold. The resonances below that threshold are associated with dipole allowed transitions from the $1 s{ }^{2} S$ ground level to $n p{ }^{2} P_{1 / 2,3 / 2}$ terms. The cross section was convoluted with a 100-meV full-width-at-half-maximum Gaussian to simulate a possible experiment.

ions, however, are the topic of the next subsection 3.2.

Given the precision of present atomic structure calculations for the hydrogen atom and for hydrogenlike ions, very accurate cross sections for photoabsorption can be obtained for these one-electron systems. Using the formalism described in the previous section the total photoabsorption cross section for $\mathrm{He}^{+}(1 s)$ ions has been determined in the frame of the present overview. From tabulated oscillator strengths and transition rates for hydrogenlike atoms and ions [57] one can construct the photoexcitation part of the total cross section using equation 5. The first 35 transitions $1 s \rightarrow n p$ with $n=2,3, \ldots, 36$ were considered. Since the excited states are all doublets $\left(n p{ }^{2} P\right)$, the oscillator strengths given in Ref. [57] have to be partitioned with ratios 1 : 2 for the ${ }^{2} P_{1 / 2}$ and ${ }^{2} P_{3 / 2}$ levels, respectively. The natural width of each fine-structure component of the strongest absorption peak $(n=2)$ is $\Gamma=6.60 \mu \mathrm{eV}$ which is small compared to the fine-structure splitting of $726 \mu \mathrm{eV}$ [58]. The total $2 p$ resonance strength $\bar{\sigma}_{1 s \rightarrow 2 p}=45.69 \mathrm{MbeV}$ is distributed among the ${ }^{2} P_{1 / 2}$ and ${ }^{2} P_{3 / 2}$ levels also with the ratio 1 : 2 , respectively.

As a result, the $2 p{ }^{2} P_{1 / 2}$ and $2 p{ }^{2} P_{3 / 2}$ resonance peaks reach heights of approximately $1.5 \times 10^{6} \mathrm{Mb}$ and $3 \times 10^{6} \mathrm{Mb}$, respectively. This is about 6 orders of magnitude above the cross section for direct $1 s$ photoionization. Even the best energy resolution at synchrotron radiation facilities which is of the order of $1 \mathrm{meV}$ would not allow one to determine the natural width $\Gamma$ of such narrow resonances $(\Gamma=6.60 \mu \mathrm{eV}$ for the $\mathrm{He}^{+}\left(2 p^{2} P_{1 / 2,3 / 2}\right)$ terms $)$. For the present purpose the Lorentzian peaks obtained from Eq. 5 were convoluted with a 100-meV full-width-at-half-maximum (FWHM) Gaussian, thus reducing the maximum of the $1 s \rightarrow 2 p$ peak cross section to about $430 \mathrm{Mb}$ (see Fig. 6). With increasing $n$ the resonance strengths of the absorption peaks very rapidly 


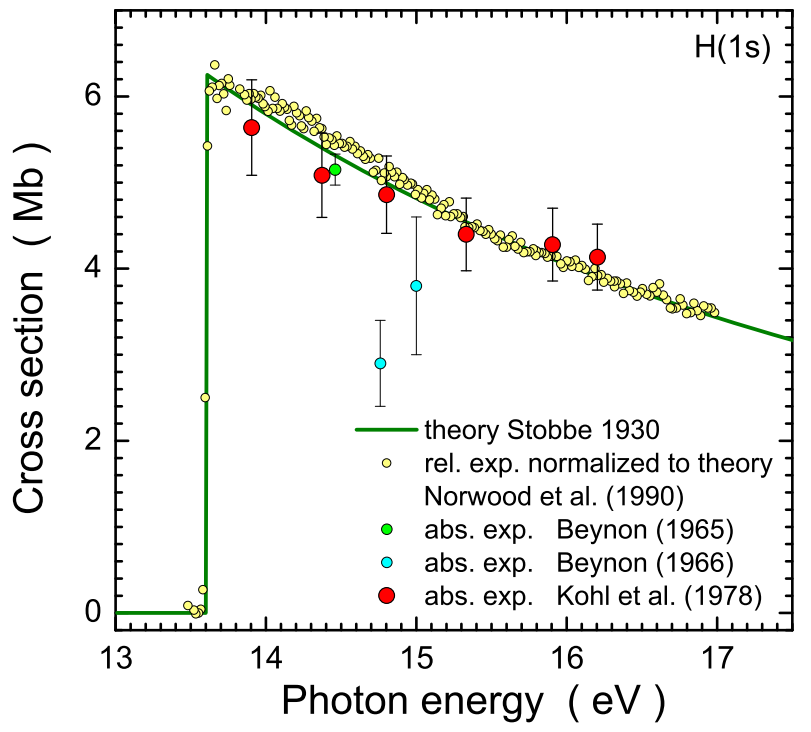

Figure 7. (Colour online) Cross section for photoionization of ground-state hydrogen atoms. The solid (green) line represents Stobbe's theory [42] and was computed using Eq. 6. The small (yellow online) shaded circles are from a relative measurement [59] which was normalized to theory. The (blue online) outliers and the (green online) data point with the smallest error bars are absolute measurements by Beynon [60,61]. The remaining six (red online) data points close to theory are absolute data taken by Kohl et al [62].

decrease (with $n^{-3}$ for sufficiently high principal quantum numbers $n$ ) and therefore, high- $n$ states add relatively little to the absorption cross section which levels off towards the photoionization threshold at $54.4178 \mathrm{eV}$. Above the threshold the photoionization cross section calculated by using Eq. 6 jumps to about $1.6 \mathrm{Mb}$. The calculated photoionization cross section was also convoluted with a 100-meV FWHM Gaussian. The resulting photoabsorption cross section is shown in Fig. 6. The two contributions, photoexcitation and photoionization, are shown separately and were not added. Typical experiments looking at just the production of photoions can only measure the nonresonant part, while an experiment based on the observation of fluorescence would only detect the resonances. With a $\mathrm{He}^{+}$target provided as an ion beam or an ion cloud stored in an ion trap, the target thickness that can be achieved is too small to observe the whole photoabsorption curve directly. Dual-plasma techniques, however, might be capable of providing sufficient column densities for direct absorption measurements with hydrogenlike ions $[4,8]$.

The accuracy of photoabsorption cross-section measurements cannot be compared with the achievements reached in the determination of wavelengths. It can even not challenge the accuracy of Stobbe's calculations for the lighter systems. However, by using the time-inversed process of radiative recombination one can study fine aspects of photoionization in collisions of fast heavy few-electron ions with gas molecules which serve to provide targets of electrons [63]. Since very highly charged ions can be probed 
by such measurements the non-relativistic calculations by Stobbe are no longer adequate and more sophisticated approaches have to be employed (see e.g. [43]).

Direct measurements on photoionization of hydrogenlike systems are very limited since the cross sections for these processes rapidly drop in size as the atomic number is increased. Presently, there are no direct photoionization measurements available for one-electron systems beyond $\mathrm{He}^{+}$. For ground-state atomic hydrogen, there are a few measurements $[59,60,61,62]$ of the photoionization cross section which is expected to be described well by Eq. 6. Fig. 7 shows the existing experimental data which illustrate the difficulty of obtaining absolute cross sections even for the simplest atom. The measurements by Beynon were probably influenced by the presence of metastable $\mathrm{H}(2 s)$ atoms produced in the $\mathrm{RF}$ gas discharge used to dissociate $\mathrm{H}_{2}$.

The experimental situation is much better for $\mathrm{H}$-like $\mathrm{He}^{+}$since these ions are magnetically analyzed and therefore possible $\mathrm{He}^{+}(2 s)$ contaminations are quenched. Moreover, the measurement of beam-overlap formfactors [64] is a fundamentally sound procedure and, if properly performed, more robust than the determination of target thickness in an effusive molecular beam of hydrogen. However, measurements of cross sections for light hydrogenlike ions can only serve the purpose of demonstrating the validity of experimental procedures employed in the typical photon-ion merged-beam arrangements: there is no reason to doubt the accuracy of the theoretical data at the level of $10-20 \%$ uncertainties typical for present state-of-the-art experiments. Only the Aarhus photon-ion study group together with their collaborators have published results for photoionization of $\mathrm{He}^{+}[65,66]$ to show the quality and accuracy of their absolute measurements. Comparable results have been obtained at the ALS but were not published. While theory must be considered superior to experiment in the determination of photoionization cross sections and threshold energies for light hydrogenlike ions, this situation might change if ensembles of very highly charged hydrogenlike heavy ions could be exposed to very-narrow-bandwidth short-wavelength radiation either in suitable traps or in heavy-ion storage rings. So far, no synchrotron radiation facility has been connected to a storage ring capable of providing beams of very highly charged H-like ions.

\subsection{He-like ions}

While photoionization experiments presently cannot help much with providing new advances in the physics of hydrogenlike ions, the situation changes immediately when more than one electron is present and when electron-electron correlations come into play. Again, as in Fig. 1, single-photon single excitation and direct single ionization are possible. In addition, single-photon double excitation of the ground state or single excitation of a $1 s 2 s$ metastable parent ion (see Fig. 2) produce an autoionizing intermediate state which can decay by an Auger process. As demonstrated by Fig. 6, direct $K$-shell ionization has a relatively small cross section in comparison with the strongest resonances. This is not only true for H-like systems but for many-electron ions 


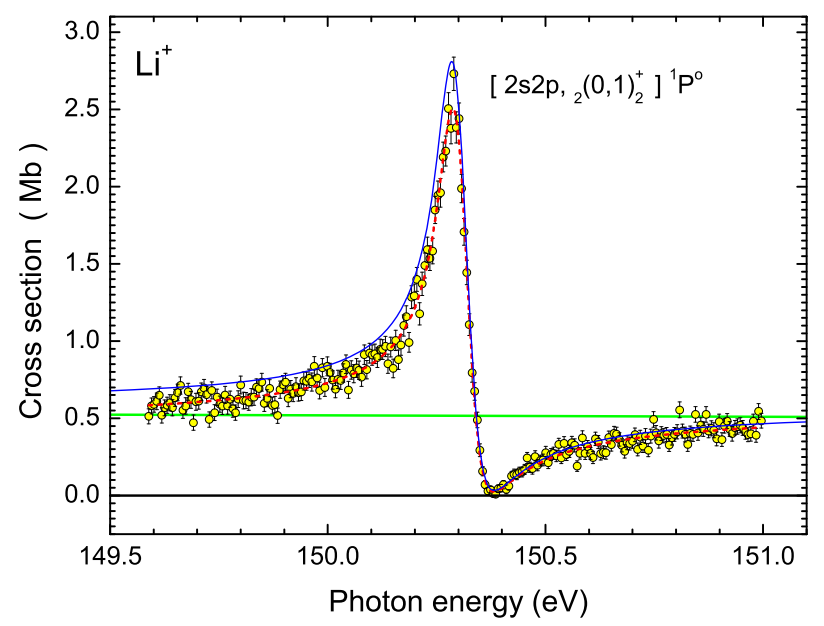

Figure 8. (Colour online) Resonance feature in the photoionization of ground-state $\mathrm{Li}^{+}$associated with the doubly excited configuration $2 s 2 p$ or, more adequate for a doubly excited two-electron system, ${ }_{2}(0,1)_{n}^{+}$, using the notation ${ }_{\mathrm{N}}(\mathrm{K}, \mathrm{T})_{n}^{\mathrm{A}}$, where $\mathrm{K}, \mathrm{T}$ and $\mathrm{A}$ are correlation quantum numbers [67,68] defined in hyperspherical coordinates. The light (yellow online) shaded circles show experimental data [12] with their statistical uncertainties. The almost horizontal (green) line represents the cross section for direct $K$-shell photoionization. It is obtained from a fit formula provided by Verner et al [47] in the frame of the opacity project. The dashed (red) line is a fit to the experimental data applying Gaussian convolution to a Fano profile according to Eq. 13 [69]. The solid blue line is the result of an R-matrix calculation [12] convoluted with a $41.4 \mathrm{meV}$ Gaussian to simulate the experimental photon-energy bandwidth in this particular measurement.

as well. Direct double excitation is a higher-order process and, hence, also has relatively small cross sections. Double excitation was seen in the photoabsorption spectrum of $\mathrm{Li}^{+}$ ions recorded by using the dual-laser-plasma technique $[4,6,7,8]$. A later experiment using the photon-ion merged-beam technique has provided absolute cross sections and detailed results for resonance parameters relevant to single-photon double excitation of ground-state $\mathrm{Li}^{+}$ions by observing net single ionization [12].

Fig. 8 shows part of the photoionization spectrum of $\mathrm{Li}^{+}\left(1 s^{2}{ }^{2} S\right)$ [12]. The resonance is associated with the $\left(2 s 2 p,{ }_{2}(0,1)_{n}^{+}\right){ }^{1} P^{o}$ level of the doubly excited $\mathrm{Li}^{+}$ion. The expected cross section for direct $K$-shell photoionization, undisturbed by resonances, is given by the almost horizontal (green) solid line which is the same as that displayed in Fig. 3. The thin (blue) line represents an R-matrix calculation which treats direct and resonance contributions on equal footing in a unified manner. The close-coupling theory reproduces the shape of the cross section remarkably well. Two ionization mechanisms interact in the present energy range around the $2 s 2 p$ resonance. Their pathways are described by the arrows in Fig. 3. Strong destructive interference is indicated by the dip at about $150.38 \mathrm{eV}$ where the two amplitudes of direct and two-step ionization totally cancel one another so that the cross section drops to zero.

The natural width $\Gamma$ of the resonance was determined by a Gauss-Fano fit (shown 


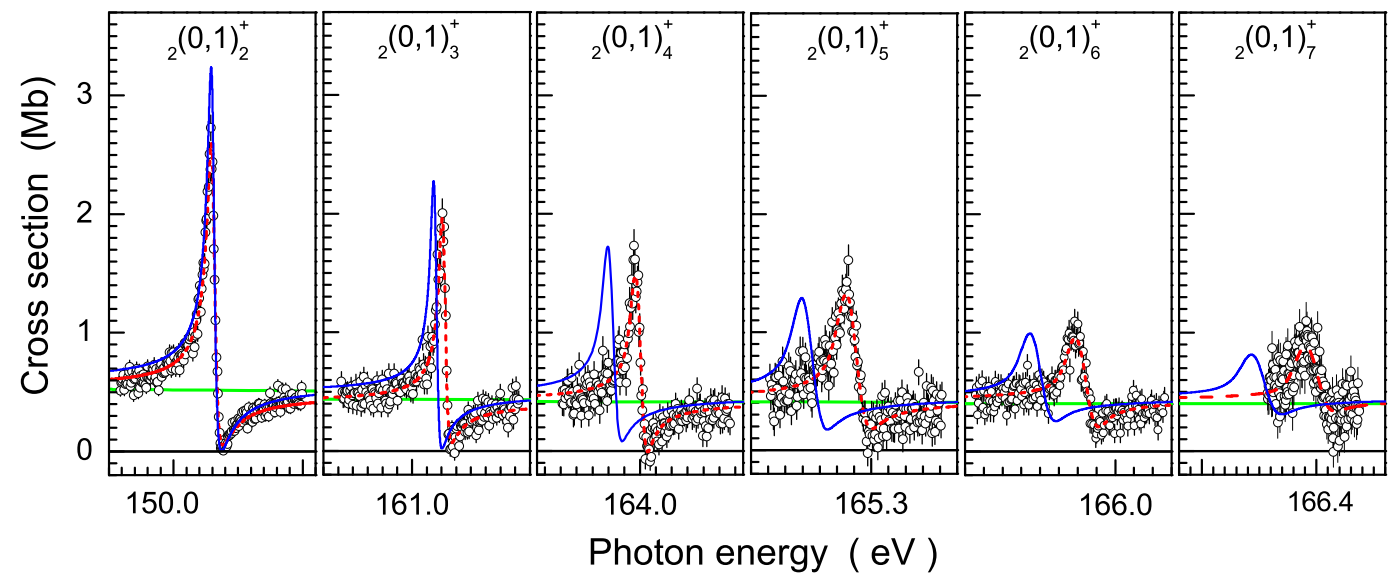

Figure 9. (Colour online) Resonances in the photoionization of ground-state $\mathrm{Li}^{+}$ associated with the sequence of doubly excited levels $2 s n p{ }^{1} \mathrm{P}^{o}$ of the $\mathrm{Li}^{+}$ion with $n=2,3, \ldots, 7[12]$. The explanations of symbols and lines is the same as in Fig. 8.

as a dashed red line in Fig. 8) to be $57 \pm 3 \mathrm{meV}$, roughly 4 orders of magnitude larger than the width of the singly-excited $2 p^{2} P$ state in $\mathrm{H}_{-}$-like $\mathrm{He}^{+}$(see subsection 3.1). This increase in natural width reflects the contribution of Auger processes to the decay of doubly excited heliumlike states $\left(\Gamma=\Gamma^{(r)}+\Gamma^{(\text {Auger })}\right)$ which typically have high transition rates for low orbital momentum quantum numbers. When the width of a resonance increases while the resonance strength stays the same, the peak cross section has to decrease proportionally. The size of the peak cross section of the $\left(2 s 2 p,{ }_{2}(0,1)_{n}^{+}\right){ }^{1} P^{o}$ resonance is close to $3 \mathrm{Mb}$, i.e., more than 6 orders of magnitude down from that of $1 s \rightarrow 2 p$ photoexcitation of $\mathrm{He}^{+}(1 s)$. The reason for this additional reduction is in the double excitation involved in populating the $\mathrm{Li}^{+}(2 s 2 p)$ resonance compared to the single excitation in $\mathrm{H}$-like $\mathrm{He}^{+}$.

In the experiment by Scully et al [12] several members of the $2 s n p$ sequence $(n=2, \ldots, 7)$ of doubly-excited resonances were investigated with a resolution of $10.7 \mathrm{meV}$ which corresponds to a resolving power $E_{\gamma} / \Delta E_{\gamma} \approx 15,000$, i.e., about 10 times better than the typical resolution of the dual-plasma experiments. The experimental results are shown in Fig. 9. With increasing principal quantum number $n$ of the Rydberg electron the theoretical resonance positions diverge from the experiment towards lower energies. The strongest deviation is observed for the ${ }_{2}(0,1)_{7}^{+}$resonance, however, it amounts to only about $24 \mathrm{meV}$. Although this is within the experimental uncertainty of $\pm 30 \mathrm{meV}$, the trend of the calculated resonance positions is obviously not fully compatible with the experimental results.

While most of the photon-ion experiments have focussed on the detection of photoionized ions there is a more recent development to also look at fluorescence subsequent to photoexcitation. For ions trapped in an EBIT this is the most common observation channel ([33] and references therein). In a recent series of experiments $K$ shell excitation of highly charged iron ions was investigated by exposing the charge-state 


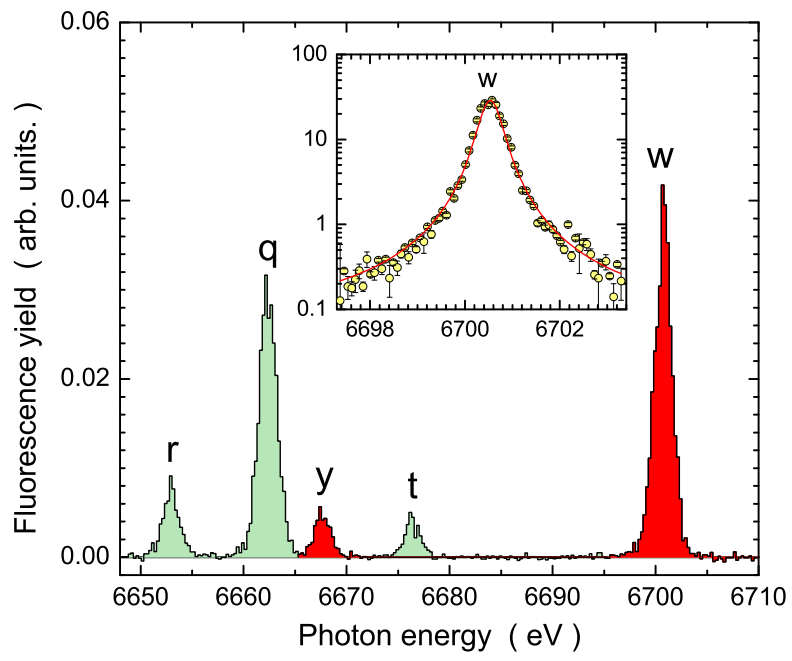

Figure 10. (Colour online) Part of an EBIT fluorescence-yield spectrum excited by photons from PETRA III. The labeling of peaks follows the notation introduced by Gabriel [70]. The dark (red) shaded peaks are associated with $\mathrm{Fe}^{24+}$, the lighter (green) shaded peaks belong to $\mathrm{Fe}^{23+}$. The spectrum shown here was measured with linearly polarized synchrotron radiation in the direction perpendicular to both the photon beam and its polarization direction. The inset displays a high-resolution measurement of the $\mathrm{w}$ line. The solid curve (red online) is a Voigt fit to the experimental data.

inventory of an EBIT to linearly polarized synchrotron radiation at PETRA III [34]. Although several ion charge states are simultaneously present in an EBIT the resolution of the experiments was sufficiently high to separate and identify the excited states of $\mathrm{Fe}^{q+}$ ions with $q=17, \ldots, 24$. A detail of the measured overview spectrum is shown in Fig. 10. The peaks labeled r, q, and t are associated with $1 s^{2} 2 s \rightarrow 1 s 2 s 2 p$ transitions in Li-like $\mathrm{Fe}^{23+}$. Peaks y and $\mathrm{w}$ are due to $1 s^{2} \rightarrow 1 s 2 p$ excitations of ground-state He-like $\mathrm{Fe}^{24+}$. The y line results from the forbidden ${ }^{3} P_{1}-{ }^{1} S_{0}$ transition, the $\mathrm{w}$ resonance line from the dipole-allowed ${ }^{1} P_{1}-{ }^{1} S_{0}$ transition. The x-ray spectra were measured with two detectors observing fluorescence perpendicularly to the photon beam axis at two observation angles of $0^{\circ}$ and $90^{\circ}$ relative to the plane defined by the polarization-direction and the photon beam.

The overview spectrum was measured at moderate resolution in order to maintain a strong signal level. The individual lines were then also scanned with significantly reduced photon-beam bandwidth while the trapped ion ensemble was evaporatively cooled in order to minimize Doppler broadening. The energy of the synchrotron radiation was carefully calibrated using absorption edges of different metals. The inset of Fig. 10 shows the precision measurement of the $\mathrm{w}$ line in He-like $\mathrm{Fe}^{24+}$. A Voigt fit provided the three main resonance parameters. The Gaussian width was determined to be $(0.38 \pm 0.06) \mathrm{eV}$ which corresponds to a resolving power of almost 18,000. The Lorentzian width was found to be $(290 \pm 30) \mathrm{meV}$. The resonance energy from the fit is $(6700.549 \pm 0.005) \mathrm{eV}$ with a statistical uncertainty of only $5 \mathrm{meV}$. The uncertainty of the energy calibration was $\pm 70 \mathrm{meV}$ resulting in a total uncertainty (one standard deviation) of the line position 

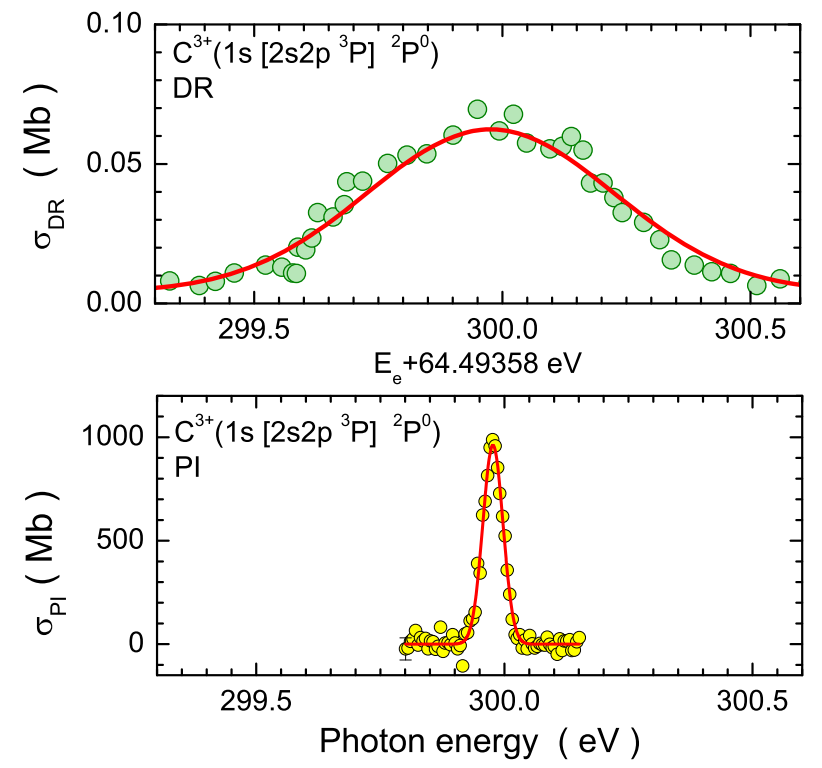

Figure 11. (Colour online) lower panel: photoionization (PI) of $\mathrm{C}^{3+}$ measured by Müller et al [17], (yellow) shaded circles; Voigt fit to the data points, solid (red) curve; upper panel: $\mathrm{C}^{4+}$ dielectronic recombination (DR) measured by Mannervik et al [72], (green) shaded circles; DR inferred from a fit to the $\mathrm{C}^{3+}\left[1 s\left(2 s 2 p^{3} \mathrm{P}\right]^{2} \mathrm{P}\right.$ photoionization resonance (shown in the lower panel), (red) solid line. The electronenergy axis is shifted by the $\mathrm{C}^{3+}$ ionization potential [58].

at the level of $10 \mathrm{ppm}$. This low uncertainty places this measurement among the most precise observations to date of resonance-line energies in heliumlike ions which test (and confirm) quantum electrodynamics (QED) calculations (see, e.g., [71]) at a very sensitive level. This is important in the light of the unexplained discrepancy between two independent measurements of the proton radius [52]. Doubts were raised concerning the QED calculations by the findings of precision spectroscopy of muonic hydrogen versus conventional electron scattering experiments from which the radii of nuclei and also the proton have been determined in the past.

Beside ground-state heliumlike ions, metastable excited ions in the relatively long lived $1 s 2 s{ }^{3} S$ state are also possible candidates for photoabsorption experiments (see Fig. $2(\mathrm{~b})$ ). In the case of $\mathrm{C}^{4+}$ parent ions spectroscopic measurements of intermediate hollow ions with 2 vacancies in the K-shell were performed [39]. This was made possible by producing a beam of $\mathrm{C}^{4+}$ containing a significant fraction of ions in the metastable $1 s 2 s^{3} S_{1}$ level. Single excitation of such an ion, which already carries an excitation energy of almost $300 \mathrm{eV}$ into the interaction with a photon, results in a double-K-vacancy state which then decays by a single-Auger process (see Fig. 2 (c)).

\subsection{Three-electron ions}

Lithiumlike ions in collision experiments employing ion beams are typically in their ground state. Since there is already an electron in the $n=2$ shell single excitation of 


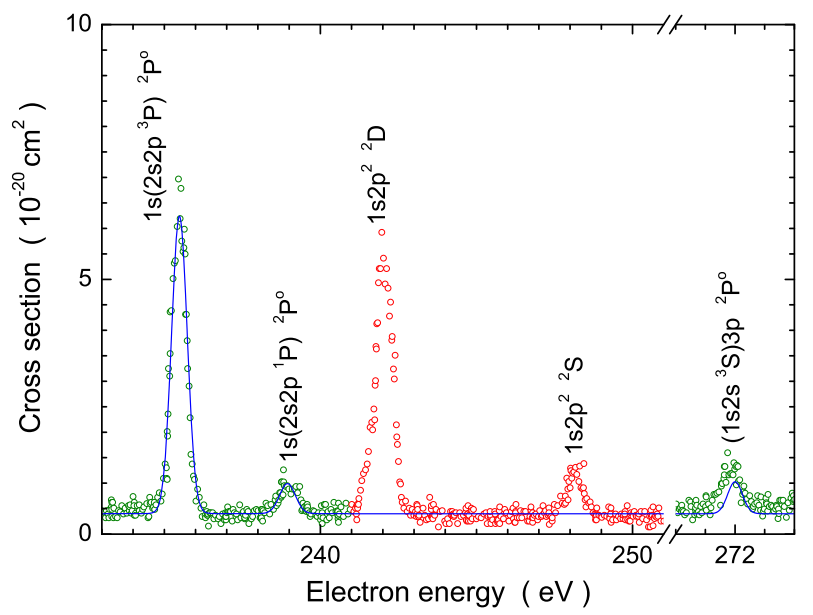

Figure 12. (Colour online) $\mathrm{C}^{4+}$ dielectronic recombination (DR); cross sections measured by Mannervik et al [72], open circles; DR cross sections inferred from electric dipole allowed transitions in the photoionization of ground-state $\mathrm{C}^{3+}$, (blue) solid line. The resonances indicated by (red) circles in the energy range 242 to $251 \mathrm{eV}$ are not accessible via single electric dipole (E1) transitions from the ground level of $\mathrm{C}^{3+}$.

a $K$-shell electron produces an autoionizing state as depicted in Fig. 4 (a). In addition to radiative relaxation similar to the transition shown in Fig. 1 (c) Auger decay is also possible as illustrated in Fig. 4 (b). As an example for the existing experimental data obtained for lithiumlike ions, the lower panel of Fig. 11 displays the $\mathrm{C}^{3+}\left(1 s\left[2 s 2 p^{3} \mathrm{P}\right]{ }^{2} \mathrm{P}^{o}\right)$ resonance which dominates the photoabsorption spectrum of $\mathrm{C}^{3+}\left(1 s^{2} 2 s^{2} S_{1 / 2}\right)$ by far [17]. The experimental energy resolution was determined from a Voigt fit of the experimental data to be $46 \mathrm{meV}$. The peak was found at a calibrated energy $E_{\gamma}=(299.98 \pm 0.03) \mathrm{eV}$. As Fig. 11 shows, the peak cross section for the photoionization process

$$
\gamma+\mathrm{C}^{3+}\left(1 s^{2} 2 s{ }^{2} S\right) \rightarrow \mathrm{C}^{3+}\left(1 s 2 s 2 p^{2} P\right) \rightarrow \mathrm{C}^{4+}\left(1 s^{2}{ }^{1} S\right)+e-64.49 \mathrm{eV}
$$

is about $1000 \mathrm{Mb}$ at the energy resolution of this experiment. The energy defect in Eq. 14 corresponds to the ionization potential of the $\mathrm{C}^{3+}\left(1 s^{2} 2 s^{2} S_{1 / 2}\right)$ ground level.

Time reversal of photoionization as described by Eq. 14 results in photorecombination via

$$
\mathrm{C}^{4+}\left(1 s^{2}{ }^{1} S\right)+e \rightarrow \mathrm{C}^{3+}\left(1 s 2 s 2 p^{2} P\right) \rightarrow \mathrm{C}^{3+}\left(1 s^{2} 2 s{ }^{2} S\right)+\gamma+64.49 \mathrm{eV}
$$

In the energy range of Fig. 11 the identical intermediate $\mathrm{C}^{3+}\left(1 s\left[2 s 2 p^{3} \mathrm{P}\right]^{2} \mathrm{P}^{o}\right)$ resonance state is populated by photoionization of $\mathrm{C}^{3+}$ as well as by dielectronic capture starting from $\mathrm{C}^{4+}$ parent ions. Dielectronic recombination of $\mathrm{C}^{4+}$ has been measured by Mannervik et al at the ion storage ring in Stockholm (CRYRING) [72]. The upper panel of Fig. 11 shows the narrow energy range of the measured spectrum where the $\mathrm{C}^{3+}\left(1 s\left[2 s 2 p^{3} \mathrm{P}\right]^{2} \mathrm{P}^{o}\right)$ resonance is located. As expected from energy conservation and the formalism described in Sec. 2 the resonance occurs at an electron energy $E_{e}=E_{\gamma}-I$ where $I=64.49358 \mathrm{eV}$ is the ionization potential of ground-state $\mathrm{C}^{3+}$ [58]. The (red) 
solid line in the upper panel of Fig. 11 was obtained from the absolute photoionization cross section measurement by applying the principle of detailed balance. The Voigt fit of the the photoionization data shown in the lower panel of Fig. 11 provided the peak area (= resonance strength). Using Eq. 10 the resonance strength for the associated dielectronic recombination was inferred. In order to match the energy resolution of the recombination experiment, the resulting dielectronic recombination cross section was convoluted with a $520 \mathrm{meV}$ FWHM Gaussian. The final result is shown by the (red) solid line in the upper panel of Fig. 11. The resonance strengths of measured and inferred recombination differ by not more than $7 \%$ indicating the high quality and consistency of both absolute measurements which used totally different experimental arrangements and procedures.

While Fig. 11 shows only one resonance, Fig. 12 comprises a wider energy range. Experimental data taken for dielectronic recombination of $\mathrm{C}^{4+}$ by Mannervik et al [72] are compared with cross sections inferred from the photoionization experiment of Müller et al [17]. The three $K$-shell photoionization resonances present in the photon energy range between about $299 \mathrm{eV}$ and $337 \mathrm{eV}$ provide information about three dielectronic recombination resonances in the electron energy range $233 \mathrm{eV}$ to $273 \mathrm{eV}$. Photoionization and photorecombination results are consistent with one another, however, there are two more resonances in dielectronic recombination which do not exist in the related $\mathrm{C}^{3+}$ photoionization energy region. The reason is that the two recombination resonances $\mathrm{C}^{3+}\left(1 s 2 p^{2}{ }^{2} D\right)$ and $\mathrm{C}^{3+}\left(1 s 2 p^{2}{ }^{2} S\right)$ decay to an excited state of the $\mathrm{C}^{3+}$ ion, namely, $\mathrm{C}^{3+}\left(1 s^{2} 2 p^{2} \mathrm{P}\right)$. The photoionization measurements, however, could only be carried out for ground-state $\mathrm{C}^{3+}\left(1 s^{2} 2 s{ }^{2} S\right)$ and, therefore, the $\mathrm{C}^{3+}\left(1 s 2 p^{2}{ }^{2} S\right)$ and $\mathrm{C}^{3+}\left(1 s 2 p^{2} D\right)$ resonances were not accessible via dipole-allowed photoexcitation. This example shows that great care is needed when comparing the results of photoionization and photorecombination experiments on the basis of the principle of detailed balance. In most cases the two groups of experiments address processes involving different sets of initial and final states not associated with one another by time reversal and, hence, detailed balance is not applicable as it is the case with the ${ }^{2} D$ and ${ }^{2} S$ resonances in the present example. In principle, branching ratios for decay processes have to be considered when applying Eq. 10 to measurements. For example, the $\mathrm{C}^{3+}\left(1 s 2 p^{2}{ }^{2} S\right)$ intermediate state may decay by a two-electron M1 transition to the ground state $\mathrm{C}^{3+}\left(1 s^{2} 2 s{ }^{2} S\right)$. The oscillator strength for such a transition is expected to be many orders of magnitude smaller than that for the E1 transitions to excited states. The associated small branching ratio prevents present state-of-the-art experiments from detecting the transition in photoionization of the $\mathrm{C}^{3+}$ ground-state.

As indicated in Fig. 10 EBIT measurements also provided information on photoabsorption by lithiumlike $\mathrm{Fe}^{23+}$ ions [34]. In addition to the observation of fluorescence the production of photoions was also monitored [35]. By combining all the experimental information about transition rates, natural linewidths and alignment in the photoexcitation of $\mathrm{Fe}^{23+}$ with linearly polarized photons, absolute radiative and Auger rates could be inferred [35] from the experiments in which relative photon and 


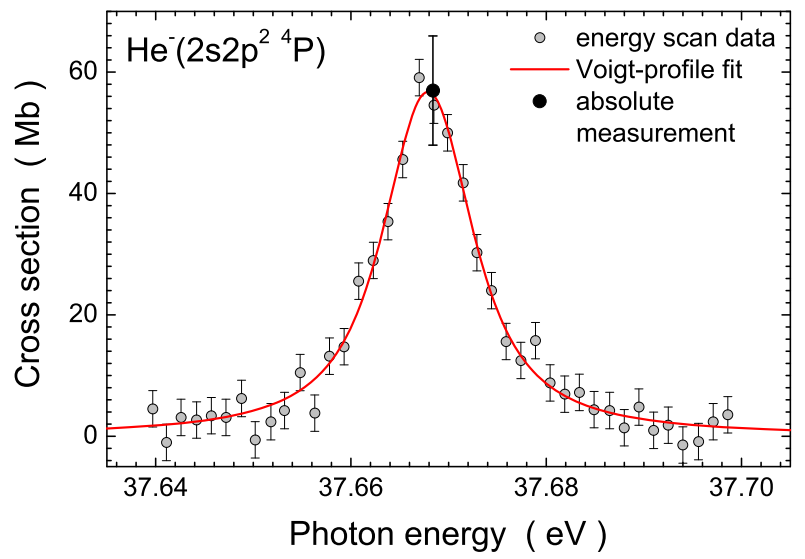

Figure 13. (Colour online) Absolute cross section for photo-double-detachment of $\mathrm{He}^{-}\left(1 s 2 s 2 p{ }^{4} P\right)$ via the intermediate triply excited $\mathrm{He}^{-}\left(2 s 2 p^{2}{ }^{4} P\right)$ term [22]. Energyscan data are shown together with their statistical error bars. On the maximum of the peak an absolute cross section was measured and is displayed as a solid circle with total error bar. The solid (red) line is a Voigt fit of the data.

photoion yields had been measured (simultaneously) rather than absolute cross sections.

Lithiumlike $\mathrm{C}^{3+}$ ions have been revisited in photoionization experiments [39] employing the new PIPE facility at DESY in Hamburg [38]. In the new experiments, the energy range of photoionization measurements was extended and the energy resolution greatly improved. For example, the $\mathrm{C}^{3+}\left(1 s\left[2 s 2 p^{3} P\right]^{2} P^{o}\right)$ resonance feature shown in the lower panel of Fig. 11 was measured with a resolving power of about 35,000. At the associated photon-beam bandwidth of less than $9 \mathrm{meV}$ it was possible to resolve the $j=1 / 2$ and $j=3 / 2$ fine-structure components of the ${ }^{2} P^{o}$ term [39].

The $\mathrm{He}^{-}\left(1 s 2 s 2 p^{4} \mathrm{P}\right)$ ion is a unique three-electron system with only one electron residing in the $K$-shell. This configuration has some similarity with that of metastable heliumlike ions in the $\left(1 s 2 s^{3} S_{1}\right)$ level. Single excitation of the $K$-shell electron produces a hollow ion with an empty $K$ shell and, in the present case, three electrons in higher shells. Previously, such hollow-state resonances had been observed in electron-impact ionization of $\mathrm{Li}^{+}$ions $[73,74]$ and in triple excitation of $\mathrm{Li}$ atoms ([4] and references therein). The triply excited states can decay by double-Auger processes resulting in net double detachment of the parent $\mathrm{He}^{-}$ion (see Fig. 4 (d) and (e) ). Resonances contributing to such double detachment have been investigated by Bilodeau et al [22]. The observed peaks can be associated with triply excited states $\left(2 s 2 p^{2}{ }^{4} P\right),\left(2 p 3 s 3 p{ }^{4} D\right)$, and $\left(2 p 3 s 3 p^{4} P\right)$. As an example, Fig. 13 displays the measured absolute cross section for photo-double-detachment of $\mathrm{He}^{-}\left(1 s 2 s 2 p^{4} P\right)$ via the intermediate triply excited $\mathrm{He}^{-}\left(2 s 2 p^{2}{ }^{4} P\right)$ term. The experiment was carried out at a photon energy bandwidth of $5.5 \mathrm{meV}$. The Voigt fit of the measured resonance yielded a natural width of $57 \mathrm{meV}$ with an uncertainty of about $15 \%$ and a resonance energy of $(37.668 \pm 0.007) \mathrm{eV}$, both numbers in fairly good to excellent agreement with several theoretical predictions already 


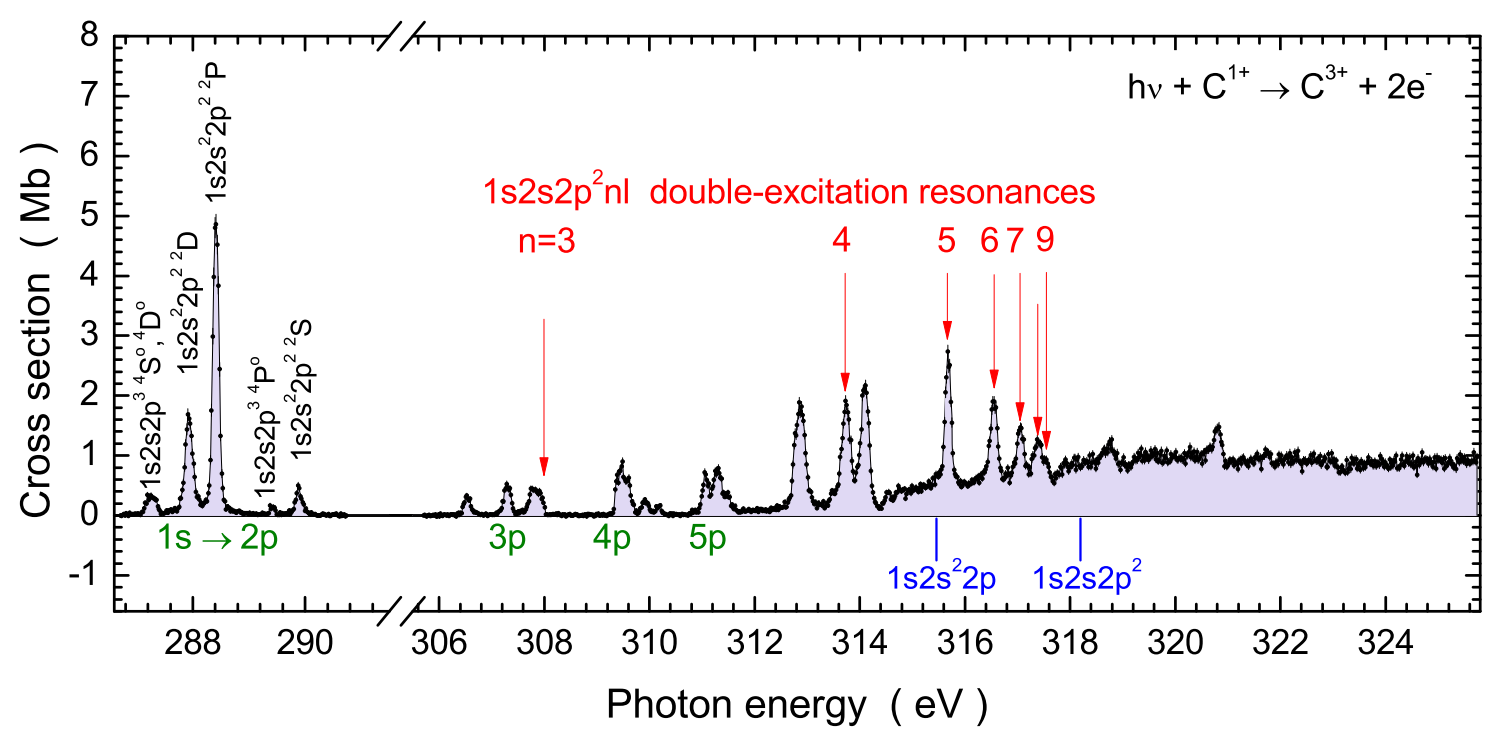

Figure 14. (Colour online) Absolute cross section for double ionization of $\mathrm{C}^{1+}$ ions by single photons [41]. The photon energy axis has a break in the range where no resonances are expected. The experimental data are shown as small dots with statistical error bars. They are connected by a solid line with shading. Important resonance groups are identified by their configurations. Thresholds for $K$-shell ionization are indicated by vertical (blue) bars.

existing at the time of the experiments.

\subsection{Many-electron ions}

When the number of electrons in the absorber is increased beyond three, more reaction pathways become accessible and the structure of photoabsorption resonances can become more complex, especially for half open outer electron shells. As already seen in negative ions, the removal of two electrons associated with $K$-shell excitation becomes possible when at least two electrons reside in higher shells above the $K$ shell. This situation is already reached with berylliumlike ions. Excitation of a $K$-shell electron produces a configuration with three electrons above a $K$ vacancy. This situation permits for example double-Auger processes to occur (see Figs. 4 (e) and 5 (e) ). For positive ions no experiments on $K$-shell excitation or ionization processes leading to net double ionization of the parent ion have been reported in the literature. Recently, complete photoionization spectra instead of isolated resonances could be measured at the new photon-ion merged-beam setup PIPE at PETRA III in Hamburg. And not only single ionization subsequent to the absorption of one photon by a $K$-shell electron was observed but also double and triple ionization [39, 40, 41].

An example of a complete double-ionization spectrum taken in the vicinity of the

$\mathrm{C}^{+} K$ edge [41] is shown in Fig. 14. The photon energies span the complete range from the energetically lowest $K$-shell excitation resonances to the region well above the $K$-shell ionization threshold. The cross section measurements are absolute with a 


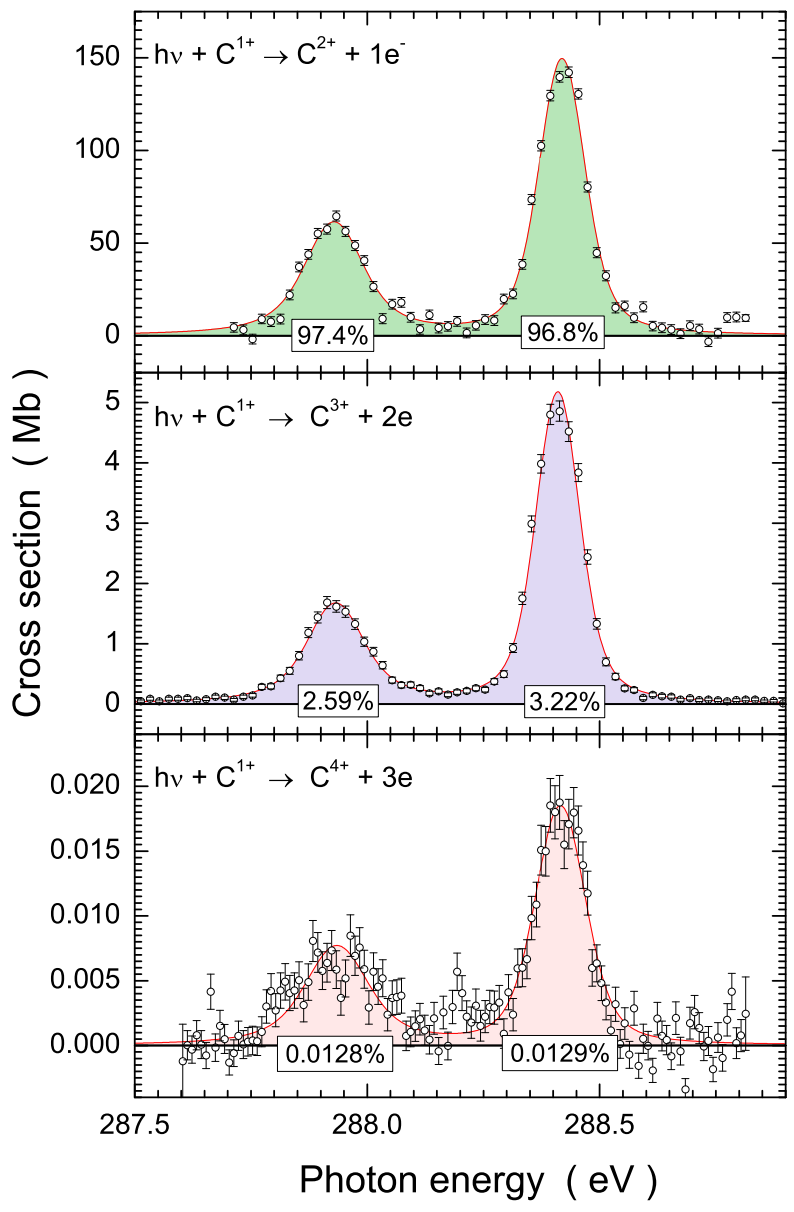

Figure 15. (Colour online) Experimental cross sections for single, double and triple ionization subsequent to $1 s-2 p$ photoexcitation of $\mathrm{C}^{+}$ions [41].

total uncertainty of about $\pm 15 \%$, the energy axis is calibrated with an uncertainty of $\pm 30 \mathrm{meV}$. Single-excitation features are indicated above the peaks. It is noted that the Rydberg sequence of resonances dominating double ionization at energies above $315 \mathrm{eV}$ is assigned to double excitations of ground-state $\mathrm{C}^{1+}$ ions to $\left(1 s 2 s 2 p^{2} n l\right)$ configurations with the principal quantum numbers $n$ indicated in Fig. 14. Resonances at still higher energies must be assigned also to single-photon double-excitation processes.

As illustrated by Fig. 5 and discussed in the last paragraph of Sec. 2 the five-electron $\mathrm{C}^{+}$ion provides the minimum number of electrons in the $L$ shell necessary for tripleAuger decay subsequent to $1 s \rightarrow 2 p$ core excitation to occur. Fig. 15 shows absolute cross section measurements for single, double and triple ionization of $\mathrm{C}^{+}$ions [41]. In the investigated energy range the following reaction pathways can occur

$$
\begin{aligned}
\gamma+\mathrm{C}^{+}\left(1 s^{2} 2 s^{2} 2 p^{2} P\right) \rightarrow \mathrm{C}^{+}(1 s & \left.2 s^{2} 2 p^{2}{ }^{2} P,{ }^{2} D\right) \\
& \rightarrow \mathrm{C}^{2+}\left(1 s^{2} 2 l 2 l^{\prime}\right)+e \\
& \rightarrow \mathrm{C}^{3+}\left(1 s^{2} 2 l\right)+2 e \\
& \rightarrow \mathrm{C}^{4+}\left(1 s^{2}\right)+3 e
\end{aligned}
$$




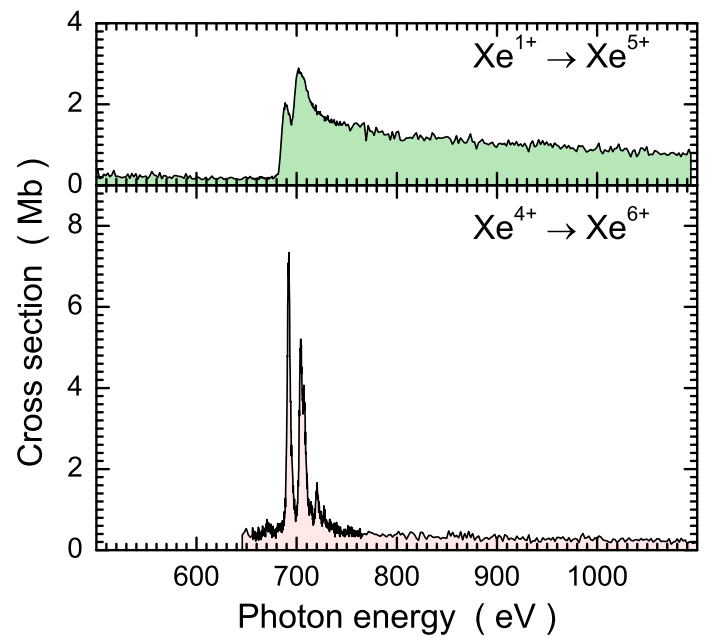

Figure 16. (Colour online) Multiple ionization of xenon ions via $3 d$-subshell photoabsorption [38]; upper panel: $\mathrm{Xe}^{+}$quadruple ionization; lower panel: $\mathrm{Xe}^{4+}$ double ionization.

Consideration of possible multi-step processes associated with two-photon absorption or a combination of single-photon excitation and a residual-gas collision cleary show that only single-Auger, double-Auger, and triple-Auger decays can explain the observations shown in the three panels of Fig. 15. With this set of measurements the first unambiguous evidence for the triple-Auger process is provided. The process involves four-electron interaction leading to the transition of one of the $L$-shell electrons to the $K$ shell while all three remaining $L$-shell electrons are simultaneously ejected into the continuum. Since the measurements yielded absolute cross sections, the branching ratios for single-, double-, and triple-Auger decays can be quantified. The numbers are given for each observed resonance in Fig. 15. Only about $10^{-4}$ of the Auger decays occur in the triple-Auger channel. It is worth noting the cross section scale of the third panel in Fig. 15. With cross sections of the order of only $5 \mathrm{~kb}$ this measurement has reached unprecedented sensitivity in the realm of photon-ion experiments.

The first successful experiments at the PIPE setup were conducted with xenon ions in charge states $q$ between 1 and 5 [38]. Fig. 16 provides a flavour of the results obtained. The upper panel shows the absolute cross section measured for quadruple ionization of $\mathrm{Xe}^{+}$ions resulting from the absorption of a single photon. The onset of the cross section is at the threshold for $3 d \rightarrow \epsilon f$ excitations. The two distinct features are due to the $3 d_{3 / 2,5 / 2}$ fine structure splitting and reflect the presence of the shape resonance that is already known from $3 d$ photoionization studies of neutral xenon. Beside quadruple ionization of $\mathrm{Xe}^{1+}\left(\mathrm{Xe}^{1+} \rightarrow \mathrm{Xe}^{5+}\right.$ as shown in the upper panel of Fig. 16) the experiment [38] also provided data for triple ionization $\left(\mathrm{Xe}^{1+} \rightarrow \mathrm{Xe}^{4+}\right)$ and 5-fold ionization $\left(\mathrm{Xe}^{1+} \rightarrow \mathrm{Xe}^{6+}\right)$. All the associated cross sections are similar to one another and can be represented by the shape of the cross section reproduced in the upper panel of Fig. 16, which was chosen for display because it covers the widest photon- 
energy range measured for the $\mathrm{Xe}^{1+} \rightarrow \mathrm{Xe}^{n+}$ transitions with $n=3,4,5$. The lower panel of Fig. 16 displays the absolute cross section for double ionization of $\mathrm{Xe}^{4+}$. Again, this measurement covers the widest energy range investigated in all the $\mathrm{Xe}^{4+} \rightarrow \mathrm{Xe}^{n+}$ transitions with $n=6,7,8,9$ (see [38]). All cross sections for parent $\mathrm{Xe}^{4+}$ ions show distinct structures with narrow resonances located at the same energies as in the example shown in Fig.16. The cross sections for $\mathrm{Xe}^{+}$and $\mathrm{Xe}^{4+}$ are strikingly different. As the charge state of the xenon ions is increased the excited-state wave function collapses and becomes bound. Accordingly, the resonances observed in (multiple) photoionization become narrow and the peak cross sections increase (see also reference [75]).

\section{Outlook}

Photon-ion interaction involving deep inner-shell excitation has become accessible at third generation synchrotron light sources that can provide sufficiently high photon energies. Recent advances in $K$-shell excitation of nitrogen and oxygen ions with subsequent autoionization have been accomplished at the new photon-ion merged-beam setup at SOLEIL which can address the photon energy range 10 to $1000 \mathrm{eV}$. While the experiments involving $K$ vacancy production by absorption of a single photon were restricted so far to single ionization via the few strongest isolated resonances, new technological advances have made it possible to study also multiple ionization and to cover a wide energy region from the energetically lowest $K$ vacancy resonances to the range far beyond the $K$ edge where multiple-electron excitations occur. Such conditions have been reached in experiments with PIPE at PETRA III. PIPE is the photon-ion merged-beam endstation of the Variable Polarization XUV beamline P04 which provides photons in the energy range $280 \mathrm{eV}$ to $3000 \mathrm{eV}$. PIPE has been optimized for low detector background and high signal rates. It accommodates experiments with ions up to very high masses reaching $q \times 50000 \mathrm{u}$ for ions $\mathrm{A}^{q+}$ at $2.4 \mathrm{keV}$ kinetic energy. Thus, not only atomic and molecular ions can be studied but also electrically charged and mass-selected clusters, biomolecules and even small nano-particles. A versatile ion-source platform allows the experimenter to use a large variety of different ion sources. Among others, an all-permanent-magnet electron-cyclotron resonance (ECR) ion source, an electrospray ion source, and a flowing-afterglow cluster ion source are available. In general, arbitrary ion sources can be accommodated. An integrated reaction microscope, a radiofrequency multipole ion trap and a high-resolution electron spectrometer together with an arrangement for collecting fluorescence photons offer bright perspectives for future experiments in the single-photon - isolated-ion regime.

Another extension of the range of possible observations was accomplished by combining an electron-beam ion trap (FLASH-EBIT) with brilliant light sources. Fluorescence and photoion production can be studied with an EBIT for ions in high charge states. The first experiments on $K$-shell excitation of F-like to He-like iron ions are promising for a frontier development of precision spectroscopy of very highly charged few-electron ions in the near future allowing for sensitive tests of quantum 
electrodynamics in strong fields.

\section{Acknowledgements}

I warmly thank Ronald A. Phaneuf, Professor emeritus of the University of Nevada, Reno, for more than thirty years of friendship and joint scientific work on interactions of ion beams with matter in the gas phase. He encouraged me to get involved in the photon-ion research work at the Advanced Light Source in Berkeley and gave me the opportunity to help develop the IPB, the Ion- $\underline{\text { Photon }}$ Beam endstation of beamline 10.0.1. that he had designed and built together with his coworkers. We can look back now to a very fruitful and enjoyable collaboration in which we jointly investigated numerous aspects of interactions of synchrotron radiation with charged particles.

I am especially grateful to Stefan E. Schippers, Adjunct Professor and member of my group in Giessen, for almost two decades of very fruitful cooperation. He has great merits in creating powerful software for analyzing data obtained in all our experiments. He joined our Berkeley experiments from the beginning and while accompanying the overall development of PIPE, the Photon-Ion spectrometer at PETRA III, he took the leading role in designing the data taking and experimental control system of mergedbeam experiments with PIPE. He also implemented the data analysis tools for PIPE and successfully demonstrated the functionality of all his developments in the first round of experiments carried out at PIPE.

I thank all the many collaborators who contributed to our joint work at the ALS IPB. Among them I am particularly indebted to Dr. A. L. David Kilcoyne, member of the ALS Scientific Support Group. He has earned my highest appreciation for his devotion to our joint scientific research and his never-ending support of our joint photonion experiments at ALS and at PETRA III.

I am very grateful to Dr. Sandor Ricz and Dr. Ticia Buhr, née Ricsóka, who joined our Giessen group for the developement and construction phase of PIPE. They contributed very substantially to the success of PIPE by developing, designing and constructing most of the PIPE hardware components that were not commercially available. I thank the whole PIPE collaboration for helping to realize the PIPE project and to make the first campaign of measurements at PIPE an overwhelming success. I am grateful to the members of the FLASH-EBIT team and thank especially PD Dr. José R. Crespo López-Urrutia for welcoming us to the recent EBIT experiments.

I gratefully acknowledge a number of grants from Deutsche Forschungsgemeinschaft (Mu 1068/10-1,2,3,4,5; Mu 1068/20-1,2; Mu 1068/22) supporting our work at the ALS over the years. My thanks also go to the German Bundesministerium für Bildung und Forschung for generously supporting the setup of PIPE and its numerous components. Participation in the recent EBIT photoabsorption experiments was made possible by grant Mu 1068/18-1 of Deutsche Forschungsgemeinschaft to S. Schippers and myself. Additional support from the Hesse State Initiative for the Development of Scientific and Economic Excellence (LOEWE) in the LOEWE-Projects ELCH and RITSAT is much 
appreciated.

\section{References}

[1] Seaton M J 1987 J. Phys. B: At. Mol. Opt. Phys 20 6363-6378

[2] Hummer D G, Berrington K A, Eissner W, Pradhan A K, Saraph H E and Tully J A 1993 Astron. Astrophys. $279298-309$

[3] Kaastra J and Paerels F (eds) 2011 High-Resolution X-Ray Spectroscopy: Past, Present and Future (Springer, Berlin)

[4] Kennedy E T, Costello J T, Mosnier J P and van Kampen P 2004 Rad. Phys. Chem. 70 291-321

[5] Mehlmann G and Esteva J M 1974 Astrophys. J. $188191-195$

[6] Carroll P K and Kennedy E T 1977 Phys. Rev. Lett. 38 1068-1071

[7] Kiernan L, Mosnier J P, Kennedy E, Costello J T and Sonntag B F 1994 Phys. Rev. Lett. 722359 $-2362$

[8] Mosnier J P, Costello J, Kennedy E and Whitty W 2000 J. Phys. B: At. Mol. Opt. Phys 335203 $-5214$

[9] Kawatsura K, Yamaoka H, Oura M, Hayaishi T, Sekioka T, Agui A, Yoshigoe A and Koike F 2002 J. Phys. B: At. Mol. Opt. Phys 35 4147-4153

[10] Yamaoka H, Oura M, Kawatsura K, Hayaishi T, Sekioka T, Agui A, Yoshigoe A and Koike F 2001 Phys. Rev. A 65012709

[11] Oura M 2010 Plasma Science and Technology 12353 - 360

[12] Scully S W J, Álvarez I, Cisneros C, Emmons E D, Gharaibeh M F, Leitner D, Lubell M S, Müller A, Phaneuf R A, Püttner R, Schlachter A S, Schippers S and McLaughlin B M 2006 J. Phys. B: At. Mol. Opt. Phys 39 3957-3968

[13] Müller A, Schippers S, Phaneuf R A, Scully S W J, Aguilar A, Cisneros C, Gharaibeh M F, Schlachter A S and McLaughlin B M 2014 J. Phys. B: At. Mol. Opt. Phys 47135201

[14] Müller A, Schippers S, Phaneuf R A, Scully S W J, Aguilar A, Cisneros C, Gharaibeh M F, Schlachter A S and McLaughlin B M 2010 J. Phys. B: At. Mol. Opt. Phys 43135602

[15] Schlachter A S, Sant'Anna M M, Covington A M, Aguilar A, Gharaibeh M F, Emmons E D, Scully S W J, Phaneuf R A, Hinojosa G, Álvarez I, Cisneros C, Müller A and McLaughlin B M 2004 J. Phys. B: At. Mol. Opt. Phys 37 L103-L109

[16] Scully S W J, Aguilar A, Emmons E D, Phaneuf R A, Halka M, Leitner D, Levin J C, Lubell M S, Püttner R, Schlachter A S, Covington A M, Schippers S, Müller A and McLaughlin B M 2005 J. Phys. B: At. Mol. Opt. Phys 38 1967-1975

[17] Müller A, Schippers S, Phaneuf R A, Scully S W J, Aguilar A, Covington A M, Álvarez I, Cisneros C, Emmons E D, Gharaibeh M F, Hinojosa G, Schlachter A S and McLaughlin B M 2009 J. Phys. B: At. Mol. Opt. Phys 42235602

[18] Gharaibeh M F, Bizau J M Cubaynes D, Guilbaud S, Hassan N El, Shorman M M Al, Miron C, Nicolas C, Robert E, Blancard C and McLaughlin B M 2011 J. Phys. B: At. Mol. Opt. Phys. 44175208

[19] Gharaibeh M F, El Hassan N, Al Shorman M M, Bizau J M, Cubaynes D, Guilbaud S, Sakho I, Blancard C and McLaughlin B M 2014 J. Phys. B: At. Mol. Opt. Phys 47065201

[20] Al Shorman M M, Gharaibeh M F, Bizau J M, Cubaynes D, Guilbaud S, Hassan N EL, Miron C, Nicolas S, Robert E, Sakho I, Blancard C and McLaughlin B M 2013 J. Phys. B: At. Mol. Opt. Phys. 46195701

[21] McLaughlin B M, Bizau J M, Cubaynes D, Al Shorman M M, Guilbaud S, Sakho I, Blancard C and Gharaibeh M F 2014 J. Phys. B: At. Mol. Opt. Phys 47115201

[22] Bilodeau R C, Bozek J D, Aguilar A, Ackerman G D, Turri G and Berrah N 2004 Phys. Rev. Lett. 93193001

[23] Bilodeau R C, Bozek J D, Ackerman G D, Aguilar A and Berrah N 2006 Phys. Rev. A 73034701 
[24] Berrah N, Bozek J D, Wills A A, Turri G, Zhou H L, Manson S T, Akerman G, Rude B, Gibson N D, Walter C W, VoKy L, Hibbert A and Ferguson S M 2001 Phys. Rev. Lett. 87253002

[25] Berrah N, Bilodeau R C, Dumitriu I, Bozek J D, Gibson N D, Walter C W, Ackerman G D, Zatsarinny O and Gorczyca T W 2007 Phys. Rev. A 76032713

[26] Walter C W, Gibson N D, Bilodeau R C, Berrah N, Bozek J D, Ackerman G D and Aguilar A 2006 Phys. Rev. A $\mathbf{7 3} 062702$

[27] Kjeldsen H, Andersen P, Folkmann F, Kristensen B and Andersen T 2001 J. Phys. B: At. Mol. Opt. Phys 34 L353-L357

[28] Andersen T 2004 Phys. Reports 394 157-313

[29] Balling P, Raarup M K, Elstrøm U V, Martinussen R, Petrunin V V and Andersen T 2007 Phys. Rev. A 76, 044701

[30] Simon M C, Epp S W, Schwarz M, Beilmann C, Schmitt B L, Baumann T M, Kubiček K, Ginzel R, Klawitter R, Mäckel V, Bernitt S, Mokler P H, Crespo López-Urrutia J R and Ullrich J 2009 J. Phys. Conf. Ser. 194012009

[31] Simon M C, Schwarz M, Epp S W, Beilmann C, Schmitt B L, Harman Z, Baumann T M, Mokler P H, Bernitt S, Ginzel R, Higgins S G, Keitel C H, Klawitter R, Kubiček K, Mäckel V, Ullrich J and Crespo López-Urrutia J R 2010 J. Phys. B: At. Mol. Opt. Phys 43065003 ISSN 0953-4075

[32] Simon M, López-Urrutia J R C, Beilmann C, Schwarz M, Harman Z, Epp S W, Schmitt B L, Baumann T M, Behar E, Bernitt S, Follath R, Ginzel R, Keitel C H, Klawitter R, Kubiček K, Mäckel V, Mokler P H, Reichardt G, Schwarzkopf O and Ullrich J 2010 Phys. Rev. Lett. 105 183001

[33] Bernitt S, Brown G V, Rudolph J K, Steinbrügge R, Graf A, Leutenegger M, Epp S W, Eberle S, Kubiček K, Mäckel V, Simon M C, Träbert E, Magee E W, Beilmann C, Hell N, Schippers S, Müller A, Kahn S M, Surzhykov A, Harman Z, Keitel C H, Clementson J, Porter F S, Schlotter W, Turner J J, Ullrich J, Beiersdorfer P and Crespo López-Urrutia J 2012 Nature 492225

[34] Rudolph J K, Bernitt S, Epp S W, Steinbrügge R, Beilmann C, Brown G V, Eberle S, Graf A, Harman Z, Hell N, Leutenegger M, Müller A, Schlage K, Wille H C, Yavaş H, Ullrich J and Crespo López-Urrutia J R 2013 Phys. Rev. Lett. 111103002

[35] Steinbrügge R, Bernitt S, Epp S W, Rudolph J K, Beilmann C, Bekker H, Eberle S, Müller A, Versolato O O, Wille H C, Yavaş H, Ullrich J and Crespo López-Urrutia J R 2014 submitted for publication

[36] Epp S, Steinbrügge R, Bernitt S, Rudolph J, Beilmann C, Bekker H, Müller A, Versolato O, Wille $\mathrm{H}$ C, Yavaş $\mathrm{H}$, Ullrich $\mathrm{J}$ and Crespo López-Urrutia $\mathrm{J}$ submitted for publication

[37] Ricsóka T, Ricz S, Schippers S, Müller A, Klumpp S, Martins M, Flesch R, Mondes V, Rühl E, Schmidt L, Dörner R, Ullrich J and Wolf A 2009 J. Phys. Conf. Ser. 194142012

[38] Schippers S, Ricz S, Buhr T, Borovik Jr A, Hellhund J, Holste K, Huber K, Schäfer H J, Schury D, Klumpp S, Mertens K, Martins M, Flesch R, Ulrich G, Rühl E, Jahnke T, Lower J, Metz D, Schmidt L P H, Schöffler M, Williams J B, Glaser L, Scholz F, Seltmann J, Viefhaus J, Dorn A, Wolf A, Ullrich J and Müller A 2014 J. Phys. B: At. Mol. Opt. Phys 47115602

[39] Müller A, Borovik Jr A, Holste K, Hellhund J, Ricz S, Viefhaus J, Klumpp S, Martins M, Kilcoyne A L D and Schippers S 2014 Photoionization of astrophysically relevant $\mathrm{C}^{q+}$ ions in the vicinity of the K-edge DESY Photon Science Annual Report 2013 User Report 20132682 (DESY, Hamburg)

[40] Müller A, Borovik Jr A, Holste K, Hellhund J, Bernhardt D, Klumpp S, Martins M, Kilcoyne A L D and Schippers S 2014 Photoionization of $\mathrm{Ne}^{q+}$ ions near the $K$-edge DESY Photon Science Annual Report 2013 User Report 20132683 (DESY, Hamburg)

[41] Müller A, Borovik Jr A, Buhr T, Hellhund J, Holste K, Kilcoyne A L D, Klumpp S, Martins M, Ricz S, Viefhaus J and Schippers S Phys. Rev. Lett., accepted for publication

[42] Stobbe M 1930 Ann. Phys. (Leipzig) 7 661-715

[43] Eichler J and Meyerhof W E 1995 Relativistic Atomic Collisions (San Diego, USA: Academic Press)

[44] Ichihara A and Eichler J 2000 At. Data Nucl. Data Tables 74 1-121 
[45] Flannery M R 2006 Electron-ion and ion-ion recombination Springer Handbook of Atomic, Molecular 83 Optical Physics ed Drake G W (New York, USA: Springer) pp 800-827

[46] Müller A 2008 Adv. At. Mol. Phys. 55 293-417

[47] Verner D A, Verland G F, Korista K T and Yakovlev D G 1996 Astrophys. J. 465487

[48] Fano U and Cooper J W 1965 Phys. Rev. 137 A1364-A1379

[49] Wehlitz R, Huang M T, DePaola B D, Levin J C, Sellin I A, Nagata T, Cooper J W and Azuma Y 1998 Phys. Rev. Lett. 81(9) 1813-1816

[50] Auger P 1925 Comptes Rendus 180, 1925, S. 65 $65-68$

[51] Carlson T A and Krause M O 1965 Phys. Rev. Lett. 14(11) 390-392

[52] Antognini A, Nez F, Schuhmann K, Amaro F D, Biraben F, Cardoso J M R, Covita D S, Dax A, Dhawan S, Diepold M, Fernandes L M P, Giesen A, Gouvea A L, Graf T, Hänsch T W, Indelicato P, Julien L, Kao C Y, Knowles P, Kottmann F, Le Bigot E O, Liu Y W, Lopes J A M, Ludhova L, Monteiro C M B, Mulhauser F, Nebel T, Rabinowitz P, dos Santos J M F, Schaller L A, Schwob C, Taqqu D, Veloso J F C A, Vogelsang J and Pohl R 2013 Science 339 $417-420$

[53] Volotka A V and Plunien G 2014 Phys. Rev. Lett. 113(2) 023002

[54] Parthey C G, Matveev A, Alnis J, Bernhardt B, Beyer A, Holzwarth R, Maistrou A, Pohl R, Predehl K, Udem T, Wilken T, Kolachevsky N, Abgrall M, Rovera D, Salomon C, Laurent P and Hänsch T W 2011 Phys. Rev. Lett. 107(20) 203001

[55] Beiersdorfer P 2010 J. Phys. B: At. Mol. Opt. Phys 43074032

[56] Volotka A V, Glazov D A, Plunien G and Shabaev V M 2013 Ann. Phys. 525636 Ú 646

[57] Wiese W L and Fuhr J R 2009 J. Phys. Chem. Ref. Data $38565-719$

[58] Kramida A E, Ralchenko Yu, Reader J and NIST ASD Team (2013) available online: http://physics.nist.gov/asd National Institute of Standards and Technology, Gaithersburg, MD

[59] Norwood K, Ng C Y and Vary J P 1990 J. Chem. Phys. 931480 - 1481

[60] Beynon J D E 1965 Nature 207405

[61] Beynon J D E 1966 Proc. Phys. Soc. $8959-61$

[62] Kohl J L, Lafyatis G P, Palenius H P and Parkinson W H 1978 Phys. Rev. A $18571-574$

[63] Stöhlker T, Ma X, Ludziejewski T, Beyer H F, Bosch F, Brinzanescu O, Dunford R W, Eichler J, Hagmann S, Ichihara A, Kozhuharov C, Krämer A, Liesen D, Mokler P H, Stachura Z, Swiat P and Warczak A 2001 Phys. Rev. Lett. 86 983-986

[64] Phaneuf R A, Havener C C, Dunn G H and Müller A 1999 Rep. Prog. Phys. 62 1143-1180

[65] Andersen P, Andersen T, Folkmann F, Ivanov V K, Kjeldsen H and West J B 2001 J. Phys. B: At. Mol. Opt. Phys 34 2009-2019

[66] Bizau J M, Champeaux J P, Cubaynes D, Wuilleumier F J, Folkmann F, Jacobsen T S, Penent F, Blancard C and Kjeldsen H 2005 Astron. Astrophys. 439 387-399

[67] Lin C D 1984 Phys. Rev. A 291019

[68] Lin C D 1986 Adv. At. Mol. Phys. 2277

[69] Schippers S 2011 Int. Rev. At. Mol. Phys. $2151-156$

[70] Gabriel A H 1972 Mon. Not. R. astr. Soc. 160 99-119

[71] Artemyev A N, Shabaev V M, Yerokhin V A, Plunien G and Soff G 2005 Phys. Rev. A 71062104

[72] Mannervik S, Asp S, Broström L, DeWitt D R, Lidberg J, Schuch R and Chung K T 1997 Phys. Rev. A 55 1810-1819

[73] Müller A, Hofmann G, Weissbecker B, Stenke M, Tinschert K, Wagner M and Salzborn E 1989 Phys. Rev. Lett. 63 758-761

[74] Müller A 2009 J. Phys. Conf. Ser. 194012002

[75] Schippers S, Borovik Jr A, Buhr T, Hellhund J, Holste K, Kilcoyne A L D, Klumpp S, Martins M, Müller A, Ricz S and Fritzsche S 2015 J. Phys. B: At. Mol. Opt. Phys submitted 